Hyperbolic Maxwell Variational Inequalities for Bean's Critical-State Model in Type-II Superconductivity

Yousept, Irwin

This text is provided by DuEPublico, the central repository of the University Duisburg-Essen.

This version of the e-publication may differ from a potential published print or online version.

DOI: https://doi.org/10.1137/16M1091939

URN: urn:nbn:de:hbz:464-20180829-083710-9

Link: https://duepublico.uni-duisburg-essen.de:443/servlets/DocumentServlet?id=46800

Legal notice:

C2017 Society for Industrial and Applied Mathematics

Source: First Published in SIAM J. Numer. Anal. in Vol. 55, No. 5, published by the Society for Industrial and Applied Mathematics (SIAM). 


\title{
HYPERBOLIC MAXWELL VARIATIONAL INEQUALITIES FOR BEAN'S CRITICAL-STATE MODEL IN TYPE-II SUPERCONDUCTIVITY*
}

\author{
IRWIN YOUSEPT ${ }^{\dagger}$
}

\begin{abstract}
This paper focuses on the numerical analysis for three-dimensional Bean's critical-state model in type-II superconductivity. We derive hyperbolic mixed variational inequalities of the second kind for the evolution Maxwell equations with Bean's constitutive law between the electric field and the current density. On the basis of the variational inequality in the magnetic induction formulation, a semidiscrete Ritz-Galerkin approximation problem is rigorously analyzed, and a strong convergence result is proven. Thereafter, we propose a concrete realization of the Ritz-Galerkin approximation through a mixed finite element method based on edge elements of Nédélec's first family, Raviart-Thomas face elements, divergence-free Raviart-Thomas face elements, and piecewise constant elements. As a final result, we prove error estimates for the proposed mixed finite element method.
\end{abstract}

Key words. hyperbolic Maxwell variational inequality, Bean's critical-state model, type-II superconductivity, mixed finite element, convergence analysis, error analysis

AMS subject classifications. 35L87, 35Q60, 65M60

DOI. $10.1137 / 16 \mathrm{M} 1091939$

1. Introduction. The physical nature of superconductivity was discovered a century ago by Heike Kamerlingh Onnes (1853-1926). He observed that the electrical resistance in mercury drops completely to zero if the temperature is cooled down below the critical temperature (4.15 $\mathrm{K}$ for mercury). This is the first fundamental property of superconductors, which in particular allows electric currents to flow in a superconductor without energy dissipation. The second fundamental nature of superconductivity was discovered by Fritz Walther Meissner (1882-1974). He found out that, being in the superconducting state at an extremely cold temperature, a superconductor does not allow any penetration of a weak magnetic field (Meissner effect). Today, superconductivity makes many new applications and key technologies possible. They include applications in magnetic resonance imaging (MRI), magnetic confinement fusion technologies, high-energy particle accelerators, magnetic levitation technologies, magnetic energy storage, and many more.

Superconductors are classified into two different types (cf. Figure 1). In type-I superconductors, the Meissner effect occurs under the condition that the temperature is below the critical one $T_{c}$ and the applied magnetic field is below some critical level $H_{c}$. Above this threshold, the Meissner effect instantly breaks down (sharp transition to normal state). Typical examples for type-I superconductors are pure metals such as aluminium, mercury, and gallium. Type-II superconductors behave completely differently from those of the first type. More precisely, they admit two different critical levels $H_{c 1}<H_{c 2}$. If the applied magnetic field is below the lower critical value $H_{c 1}$,

*Received by the editors August 31, 2016; accepted for publication (in revised form) April 26, 2017; published electronically October 12, 2017.

http://www.siam.org/journals/sinum/55-5/M109193.html

Funding: The work of the author was supported by the German Research Foundation Priority Programm DFG SPP 1962 "Non-smooth and Complementarity-based Distributed Parameter Systems: Simulation and Hierarchical Optimization", Project YO 159/2-1.

${ }^{\dagger}$ Fakultät für Mathematik, Universität Duisburg-Essen, D-45127 Essen, Germany (irwin.yousept@ uni-due.de). 


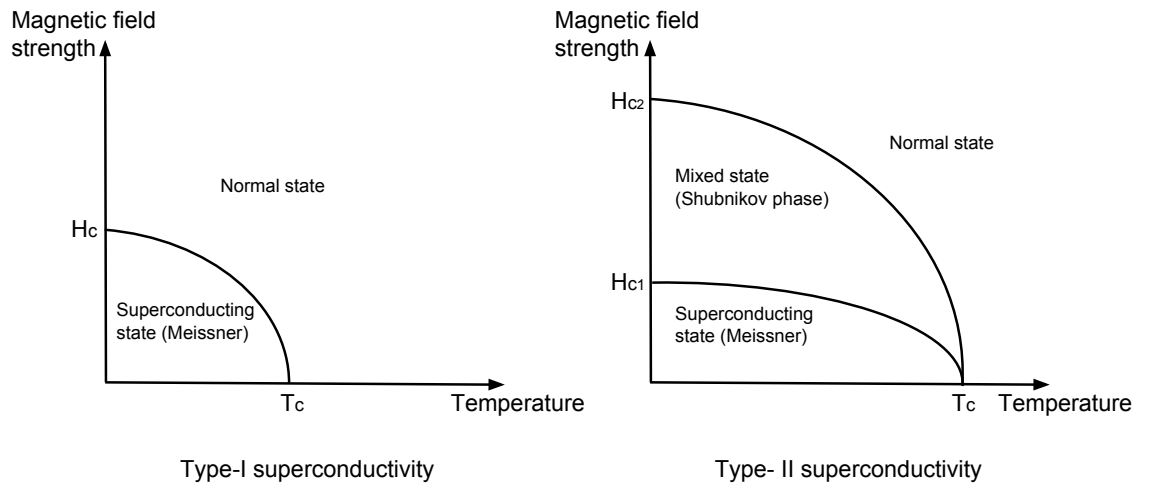

FIG. 1. Sharp transition to the normal state in type-I superconductors (left plot) and the mixed state in type-II superconductors (right plot).

then the Meissner effect occurs. If the magnetic field is stronger than $H_{c 1}$ but weaker than $H_{c_{2}}$, then the magnetic field partially enters the material, but the superconducting state is not completely destroyed. This kind of physical state is called the Shubnikov phase or mixed state. Finally, the superconducting state completely breaks down if the applied magnetic field is stronger than $H_{c 2}$. Type-II superconductors admit greater critical temperatures and critical values of magnetic field than those of the first kind. These properties enable them to preserve their superconducting effects in the presence of a strong magnetic field at higher temperatures. Examples of type-II superconductors are alloys and oxide ceramic materials.

Being in the mixed state, a type-II superconductor allows partial penetration of the applied magnetic field in the form of flux tubes. Every tube carries exactly one single magnetic flux quantum and is surrounded by a supercurrent vortex. If we modify the applied magnetic field, then the density of the flux tubes and the supercurrents will change. This dynamic magnetization process is not reversible and exhibits hysteresis. A well-known critical-state model describing such a complex irreversible magnetization process was proposed by Bean $[7,8]$. His model postulates a nonsmooth constitutive relation between the electric field and the current density as follows:

(A1) the current density strength cannot exceed some critical value $j_{c} \in \mathbb{R}^{+}$;

(A2) the electric field vanishes if the current density strength is strictly less than $j_{c}$;

(A3) the electric field is parallel to the current density.

We note that Bean made a simplifying assumption of a constant critical current density $j_{c} \in \mathbb{R}^{+}$, which is physically reasonable in the case of a not so strong magnetic field. According to experiments, however, the critical current density can depend on the magnetic field $j_{c}=j_{c}(|\boldsymbol{H}|)$ in the case of strong external fields. This physical phenomenon was observed by Kim, Hempstead, and Strnad [21]. We refer the reader to [10] for a comprehensive review on the derivation of the Bean critical-state constitutive relation from different mathematical models, including Ginzburg-Landau and London equations. See also $[11,12]$ for mathematical and numerical results on Ginzburg-Landau equations.

Let $\Omega \subset \mathbb{R}^{3}$ be a bounded Lipschitz domain and let $\Omega_{s c}$ be an open set satisfying $\bar{\Omega}_{s c} \subset \Omega$. Here, the subset $\Omega_{s c}$ represents a type-II superconductor. Assuming that the temperature of the superconductor $\Omega_{s c}$ is below the critical one, the evolution of the electromagnetic waves in $\Omega$ is described by the Maxwell equations 


$$
\begin{cases}\epsilon \boldsymbol{E}_{t}-\operatorname{curl} \boldsymbol{H}+\boldsymbol{J}=\mathbf{f} & \text { in } \Omega \times(0, T), \\ \mu \boldsymbol{H}_{t}+\operatorname{curl} \boldsymbol{E}=0 & \text { in } \Omega \times(0, T), \\ \boldsymbol{E} \times \boldsymbol{n}=0 & \text { on } \partial \Omega \times(0, T), \\ \boldsymbol{E}(\cdot, 0)=\boldsymbol{E}_{0} & \text { in } \Omega, \\ \boldsymbol{H}(\cdot, 0)=\boldsymbol{H}_{0} & \text { in } \Omega,\end{cases}
$$

along with the Bean constitutive law (A1)-(A3) for the electric field and the current density:

$$
\begin{cases}\boldsymbol{J}(x, t) \cdot \boldsymbol{E}(x, t)=g(x)|\boldsymbol{E}(x, t)| & \text { a.e. in } \Omega \times(0, T), \\ |\boldsymbol{J}(x, t)| \leq g(x) & \text { a.e. in } \Omega \times(0, T) .\end{cases}
$$

In the setting of (1.1a), $\boldsymbol{E}: \Omega \times(0, T) \rightarrow \mathbb{R}^{3}$ denotes the electric field, $\boldsymbol{H}: \Omega \times(0, T) \rightarrow$ $\mathbb{R}^{3}$ the magnetic field, $\boldsymbol{J}: \Omega \times(0, T) \rightarrow \mathbb{R}^{3}$ the current density, $\mathbf{f}: \Omega \times(0, T) \rightarrow \mathbb{R}^{3}$ the applied current source, and $\boldsymbol{E}_{0}, \boldsymbol{H}_{0}: \Omega \rightarrow \mathbb{R}^{3}$ the initial electric and magnetic fields. Furthermore, the scalar functions $\epsilon, \mu: \Omega \rightarrow \mathbb{R}$ stand for the electric permittivity and the magnetic permeability, respectively. They are of class $L^{\infty}(\Omega)$ and satisfy

$$
\underline{\epsilon} \leq \epsilon(x) \leq \bar{\epsilon} \quad \text { a.e. in } \Omega \quad \text { and } \quad \underline{\mu} \leq \mu(x) \leq \bar{\mu} \quad \text { a.e. in } \Omega,
$$

for some constants $0<\underline{\epsilon}<\bar{\epsilon}$ and $0<\underline{\mu}<\bar{\mu}$. Moreover, the scalar function $g: \Omega \rightarrow \mathbb{R}$ is assumed to be of class $L^{\infty}(\Omega)$ and nonnegative. In the context of Bean's criticalstate model, it is given by

$$
g(x)=j_{c} \chi_{\Omega_{s c}}(x),
$$

where $j_{c} \in \mathbb{R}^{+}$is the critical current density of the type-II superconductor $\Omega_{s c}$, and $\chi_{\Omega_{s c}}$ denotes the characteristic function of $\Omega_{s c}$.

If the displacement current $\epsilon \boldsymbol{E}_{t}$ is significantly smaller in comparison with $-\operatorname{curl} \boldsymbol{H}+\boldsymbol{J}$, then Maxwell's equations (1.1a) can be approximated by neglecting $\epsilon \boldsymbol{E}_{t}$. This approximation is called eddy current approximation (see [1]), which leads to a magnetic field formulation in the form of a parabolic variational inequality of the first kind. Prigozhin [26, 25] was the first to introduce and analyze this formulation. The finite element analysis in a 2D setting was investigated in [14]. Some years later, Elliott and Kashima [13] investigated the numerical analysis of the associated 3D parabolic variational inequality, where the finite element approximations of the extended Bean model by Bossavit [9] and the $\boldsymbol{E}$ - $\boldsymbol{J}$ power law were analyzed. In the case of a nonlinear critical current density $j_{c}=j_{c}(|\boldsymbol{H}|)$, the eddy current approximation of (1.1) leads to a parabolic quasi-variational inequality. Barrett and Prigozhin [4] analyzed the associated parabolic quasi-variational inequality problem in a scalar $2 \mathrm{D}$ setting and its dual formulation. Recently, they [6] introduced a nonconforming finite element method. They proved the convergence of their nonconforming method and illustrated its efficiency numerically. See also [5] concerning the mathematical and numerical analysis for a mixed formulation of a thin film magnetization problem in type-II superconductivity.

All the previously mentioned contributions are devoted to the eddy current approximation, which simplifies the Maxwell equations (1.1a) by eliminating the displacement current $\epsilon \boldsymbol{E}_{t}$. However, in many important physical phenomena such as high-frequency physics, including radio frequency and microwave physics, the displacement current $\epsilon \boldsymbol{E}_{t}$ is of significance and in general cannot be neglected. Jochmann [18, 19] was the first to introduce (1.1) and prove its existence and uniqueness of solutions. 
In [20], he extended the existence and uniqueness result to the nonlinear case $j_{c}=$ $j_{c}(|\boldsymbol{H}|)$. The optimization of (1.1) was also recently analyzed in [29] (see also [27, 28]).

This paper focuses on the numerical analysis for (1.1). To the best of the author's knowledge, there is no earlier contribution to the numerical analysis of (1.1). In this paper, we introduce a hyperbolic mixed variational inequality of the second kind and prove the equivalence between the proposed variational inequality and the nonsmooth Maxwell system (1.1). In particular, we derive a hyperbolic mixed variational inequality in the magnetic induction formulation, which serves as the key tool for our numerical analysis. Based on this formulation, a semidiscrete Ritz-Galerkin approximation is proposed and rigorously analyzed. We prove a strong convergence result through the use of a discrete mixed variational problem and the Hilbert projection theorem applied to the range of the rotation operator acting on a curl-conforming finite-dimensional subspace. Hereafter, we discuss a concrete realization of the Ritz-Galerkin approximation through mixed finite elements, including edge elements of Nédélec's first family, Raviart-Thomas face elements, divergence-free Raviart-Thomas face elements, and piecewise constant elements. For the proposed mixed finite element method, we are able to prove error estimates yielding a convergence rate of the method.

2. Preliminaries. Throughout this paper, $c$ denotes a generic positive constant that can take different values on different occasions. For a given Hilbert space $V$, we use the notation $\|\cdot\|_{V}$ and $(\cdot, \cdot)_{V}$ for the norm and the scalar product in $V$. Furthermore, a bold typeface is employed to indicate a three-dimensional vector function or a Hilbert space of three-dimensional vector functions. Our main Hilbert spaces are

$$
\boldsymbol{H}(\operatorname{curl}):=\left\{\boldsymbol{q} \in \boldsymbol{L}^{2}(\Omega) \mid \operatorname{curl} \boldsymbol{q} \in \boldsymbol{L}^{2}(\Omega)\right\} \text { and } \boldsymbol{H}(\operatorname{div}):=\left\{\boldsymbol{q} \in \boldsymbol{L}^{2}(\Omega) \mid \operatorname{div} \boldsymbol{q} \in L^{2}(\Omega)\right\},
$$

where the curl- and div-operators are understood in the sense of distributions. As usual, $\mathcal{C}_{0}^{\infty}(\Omega)$ stands for the space of all infinitely differentiable vector functions with compact support contained in $\Omega$. We denote the closure of $\mathcal{C}_{0}^{\infty}(\Omega)$ with respect to the $\boldsymbol{H}$ (curl)-topology and the $\boldsymbol{H}$ (div)-topology, respectively, by $\boldsymbol{H}_{0}(\mathbf{c u r l}):=$ ${\overline{\mathcal{C}_{0}^{\infty}(\Omega)}}^{\|\cdot\|_{\boldsymbol{H}(\mathrm{curl})}}$ and $\boldsymbol{H}_{0}(\operatorname{div}):={\overline{\mathcal{C}_{0}^{\infty}(\Omega)}}^{\|\cdot\|_{\boldsymbol{H}(\mathrm{div})}}$. Furthermore,

$$
\boldsymbol{H}_{0}(\operatorname{div}=0):=\left\{\boldsymbol{q} \in \boldsymbol{L}^{2}(\Omega) \mid(\boldsymbol{q}, \nabla \phi)_{\boldsymbol{L}^{2}(\Omega)}=0 \quad \forall \phi \in H^{1}(\Omega)\right\} .
$$

Finally, for every positive function $\alpha \in L^{\infty}(\Omega)$, we use the notation $\boldsymbol{L}_{\alpha}^{2}(\Omega)$ for the weighted $\boldsymbol{L}^{2}(\Omega)$-space endowed with the weighted scalar product $(\alpha \cdot, \cdot)_{\boldsymbol{L}^{2}(\Omega)}$.

The existence of unique mild and strong solutions to the hyperbolic system (1.1) has been proved by Jochmann in [18, Theorem 1] and [19, Lemma 4.3]. We summarize the existence and uniqueness result for the strong solution in the following lemma.

Lemma 2.1. Let $\mathbf{f} \in W^{1, \infty}\left((0, T), \boldsymbol{L}^{2}(\Omega)\right)$ and $\left(\boldsymbol{E}_{0}, \boldsymbol{H}_{0}\right) \in \boldsymbol{H}_{0}(\mathbf{c u r l}) \times \boldsymbol{H}(\mathbf{c u r l})$. Then, there exist a unique pair $(\boldsymbol{E}, \boldsymbol{H}) \in L^{\infty}\left((0, T), \boldsymbol{H}_{0}(\mathbf{c u r l}) \times \boldsymbol{H}(\mathbf{c u r l})\right) \cap W^{1, \infty}$ $\left((0, T), \boldsymbol{L}_{\epsilon}^{2}(\Omega) \times \boldsymbol{L}_{\mu}^{2}(\Omega)\right)$ and a unique $\boldsymbol{J} \in L^{\infty}\left((0, T), \boldsymbol{L}^{\infty}(\Omega)\right)$ satisfying (1.1b) and

$$
\left\{\begin{aligned}
\epsilon \frac{d}{d t} \boldsymbol{E}(t)-\operatorname{curl} \boldsymbol{H}(t)+\boldsymbol{J}(t) & =\mathbf{f}(t) \quad \text { for a.e. } t \in(0, T), \\
\mu \frac{d}{d t} \boldsymbol{H}(t)+\operatorname{curl} \boldsymbol{E}(t) & =0 \quad \text { for a.e. } t \in(0, T), \\
(\boldsymbol{E}, \boldsymbol{H})(0) & =\left(\boldsymbol{E}_{0}, \boldsymbol{H}_{0}\right) .
\end{aligned}\right.
$$

In other words, $(\boldsymbol{E}, \boldsymbol{H}, \boldsymbol{J})$ is the strong solution of (1.1). 
3. Hyperbolic variational inequalitites. We start by introducing the functional

$$
\varphi: \boldsymbol{L}^{1}(\Omega) \rightarrow \mathbb{R}, \quad \varphi(\boldsymbol{v})=\int_{\Omega} g(x)|\boldsymbol{v}(x)| d x .
$$

Since $g \in L^{\infty}(\Omega)$ is nonnegative, $\varphi$ defines a seminorm on $\boldsymbol{L}^{1}(\Omega)$. Taking the functional $\varphi$ into account, we introduce a hyperbolic mixed variational inequality of the second kind and prove the equivalence between (1.1) and the proposed variational inequality.

Theorem 3.1. Let $\mathbf{f} \in W^{1, \infty}\left((0, T), \boldsymbol{L}^{2}(\Omega)\right)$ and $\left(\boldsymbol{E}_{0}, \boldsymbol{H}_{0}\right) \in \boldsymbol{H}_{0}(\mathbf{c u r l}) \times \boldsymbol{H}(\mathbf{c u r l})$. Then, the hyperbolic mixed variational inequality

$$
\left\{\begin{array}{l}
\int_{\Omega} \epsilon \frac{d}{d t} \boldsymbol{E}(t) \cdot(\boldsymbol{v}-\boldsymbol{E}(t))+\mu \frac{d}{d t} \boldsymbol{H}(t) \cdot(\boldsymbol{w}-\boldsymbol{H}(t)) d x \\
\quad+\int_{\Omega} \operatorname{curl} \boldsymbol{E}(t) \cdot(\boldsymbol{w}-\boldsymbol{H}(t))-\boldsymbol{H}(t) \cdot \operatorname{curl}(\boldsymbol{v}-\boldsymbol{E}(t)) d x \\
\quad+\varphi(\boldsymbol{v})-\varphi(\boldsymbol{E}(t)) \geq \int_{\Omega} \mathbf{f}(t) \cdot(\boldsymbol{v}-\boldsymbol{E}(t)) d x \\
\text { for a.e. } t \in(0, T) \text { and all }(\boldsymbol{v}, \boldsymbol{w}) \in \boldsymbol{H}_{0}(\operatorname{curl}) \times \boldsymbol{L}^{2}(\Omega), \\
(\boldsymbol{E}, \boldsymbol{H})(0)=\left(\boldsymbol{E}_{0}, \boldsymbol{H}_{0}\right)
\end{array}\right.
$$

admits a unique solution $(\boldsymbol{E}, \boldsymbol{H}) \in L^{\infty}\left((0, T), \boldsymbol{H}_{0}(\mathbf{c u r l}) \times \boldsymbol{H}(\mathbf{c u r l})\right) \cap W^{1, \infty}((0, T)$, $\left.\boldsymbol{L}_{\epsilon}^{2}(\Omega) \times \boldsymbol{L}_{\mu}^{2}(\Omega)\right)$. The unique solution of (3.1) is exactly the strong solution of (1.1).

Proof. Uniqueness. Inserting $(\boldsymbol{v}, \boldsymbol{w})=0$ and $(\boldsymbol{v}, \boldsymbol{w})=(2 \boldsymbol{E}(t), 2 \boldsymbol{H}(t))$ into $(3.1)$, it follows that every solution $(\boldsymbol{E}, \boldsymbol{H}) \in L^{\infty}\left((0, T), \boldsymbol{H}_{0}(\mathbf{c u r l}) \times \boldsymbol{H}(\mathbf{c u r l})\right) \cap W^{1, \infty}((0, T)$, $\left.\boldsymbol{L}_{\epsilon}^{2}(\Omega) \times \boldsymbol{L}_{\mu}^{2}(\Omega)\right)$ of $(3.1)$ satisfies

$$
\begin{gathered}
\int_{\Omega} \epsilon \frac{d}{d t} \boldsymbol{E}(t) \cdot \boldsymbol{E}(t)+\mu \frac{d}{d t} \boldsymbol{H}(t) \cdot \boldsymbol{H}(t) d x+\varphi(\boldsymbol{E}(t)) \\
=\int_{\Omega} \mathbf{f}(t) \cdot \boldsymbol{E}(t) d x \quad \text { for a.e. } t \in(0, T)
\end{gathered}
$$

such that every solution of (3.1) satisfies

$$
\begin{gathered}
\int_{\Omega} \epsilon \frac{d}{d t} \boldsymbol{E}(t) \cdot \boldsymbol{v}+\mu \frac{d}{d t} \boldsymbol{H}(t) \cdot \boldsymbol{w} d x+\int_{\Omega} \operatorname{curl} \boldsymbol{E}(t) \cdot \boldsymbol{w}-\boldsymbol{H}(t) \cdot \operatorname{curl} \boldsymbol{v} d x+\varphi(\boldsymbol{v}) \\
\geq \int_{\Omega} \mathbf{f}(t) \cdot \boldsymbol{v} d x \quad \text { for a.e. } t \in(0, T) \text { and all }(\boldsymbol{v}, \boldsymbol{w}) \in \boldsymbol{H}_{0}(\operatorname{curl}) \times \boldsymbol{L}^{2}(\Omega) .
\end{gathered}
$$

Suppose now that $\left(\boldsymbol{E}^{1}, \boldsymbol{H}^{1}\right),\left(\boldsymbol{E}^{2}, \boldsymbol{H}^{2}\right) \in L^{\infty}\left((0, T), \boldsymbol{H}_{0}(\mathbf{c u r l}) \times \boldsymbol{H}(\mathbf{c u r l})\right) \cap W^{1, \infty}$ $\left((0, T), \boldsymbol{L}_{\epsilon}^{2}(\Omega) \times \boldsymbol{L}_{\mu}^{2}(\Omega)\right)$ are solutions to $(3.1)$. Then, the difference $(\boldsymbol{e}, \boldsymbol{h}):=\left(\boldsymbol{E}^{1}-\right.$ $\left.\boldsymbol{E}^{2}, \boldsymbol{H}^{1}-\boldsymbol{H}^{2}\right)$ fulfils

$$
\begin{array}{r}
\int_{\Omega} \epsilon \frac{d}{d t} \boldsymbol{e}(t) \cdot \boldsymbol{v}+\mu \frac{d}{d t} \boldsymbol{h}(t) \cdot \boldsymbol{w} d x+\int_{\Omega} \operatorname{curl} \boldsymbol{e}(t) \cdot \boldsymbol{w}-\boldsymbol{h}(t) \cdot \operatorname{curl} \boldsymbol{v} d x \geq 0 \\
\text { for a.e. } t \in(0, T) \text { and all }(\boldsymbol{v}, \boldsymbol{w}) \in \boldsymbol{H}_{0}(\operatorname{curl}) \times \boldsymbol{L}^{2}(\Omega) .
\end{array}
$$

Setting $(\boldsymbol{v}, \boldsymbol{w})=-(\boldsymbol{e}(t), \boldsymbol{h}(t))$ in (3.2) results in

$$
\begin{aligned}
0 & \geq \int_{\Omega} \epsilon \frac{d}{d t} \boldsymbol{e}(t) \cdot \boldsymbol{e}(t)+\mu \frac{d}{d t} \boldsymbol{h}(t) \cdot \boldsymbol{h}(t) d x \\
& =\frac{1}{2} \frac{d}{d t}\|\boldsymbol{e}(t)\|_{\boldsymbol{L}_{\epsilon}^{2}(\Omega)}^{2}+\frac{1}{2} \frac{d}{d t}\|\boldsymbol{h}(t)\|_{\boldsymbol{L}_{\mu}^{2}(\Omega)}^{2} \quad \text { for a.e. } t \in(0, T) .
\end{aligned}
$$


In view of this inequality and $\boldsymbol{e}(0)=\boldsymbol{h}(0)=0$, it follows that (3.1) has at most only one solution.

Existence. Lemma 2.1 implies the existence of a unique $(\boldsymbol{E}, \boldsymbol{H}) \in L^{\infty}((0, T)$, $\boldsymbol{H}_{0}($ curl $) \times \boldsymbol{H}($ curl $\left.)\right) \cap W^{1, \infty}\left((0, T), \boldsymbol{L}_{\epsilon}^{2}(\Omega) \times \boldsymbol{L}_{\mu}^{2}(\Omega)\right)$ and a unique $\boldsymbol{J} \in L^{\infty}((0, T)$, $\boldsymbol{L}^{\infty}(\Omega)$ ) satisfying (1.1b) and (2.1). Multiplying the first equality in (2.1) by $\boldsymbol{v}-\boldsymbol{E}(t)$, with $\boldsymbol{v} \in \boldsymbol{H}_{0}(\mathbf{c u r l})$, and the second equality in (2.1) by $\boldsymbol{w}-\boldsymbol{H}(t)$, with $\boldsymbol{w} \in \boldsymbol{L}^{2}(\Omega)$, and then integrating the resulting equalities over $\Omega$, we obtain

$$
\begin{aligned}
& \int_{\Omega} \epsilon \frac{d}{d t} \boldsymbol{E}(t) \cdot(\boldsymbol{v}-\boldsymbol{E}(t)) d x-\int_{\Omega} \operatorname{curl} \boldsymbol{H}(t) \cdot(\boldsymbol{v}-\boldsymbol{E}(t)) d x \\
& \quad+\int_{\Omega} \boldsymbol{J}(t) \cdot(\boldsymbol{v}-\boldsymbol{E}(t)) d x=\int_{\Omega} \mathbf{f}(t) \cdot(\boldsymbol{v}-\boldsymbol{E}(t)) d x \\
& \int_{\Omega} \mu \frac{d}{d t} \boldsymbol{H}(t) \cdot(\boldsymbol{w}-\boldsymbol{H}(t)) d x+\int_{\Omega} \operatorname{curl} \boldsymbol{E}(t) \cdot(\boldsymbol{w}-\boldsymbol{H}(t)) d x=0
\end{aligned}
$$

for a.e. $t \in(0, T)$. Then, applying

$$
\int_{\Omega} \operatorname{curl} \boldsymbol{z} \cdot \boldsymbol{v} d x=\int_{\Omega} \boldsymbol{z} \cdot \operatorname{curl} \boldsymbol{v} d x \quad \forall(\boldsymbol{v}, \boldsymbol{z}) \in \boldsymbol{H}_{0}(\operatorname{curl}) \times \boldsymbol{H}(\operatorname{curl})
$$

to the first equality in (3.3), and adding the resulting equality to the second equality in (3.3), it follows that

$$
\begin{array}{r}
\int_{\Omega} \epsilon \frac{d}{d t} \boldsymbol{E}(t) \cdot(\boldsymbol{v}-\boldsymbol{E}(t))+\mu \frac{d}{d t} \boldsymbol{H}(t) \cdot(\boldsymbol{w}-\boldsymbol{H}(t)) d x+\int_{\Omega} \operatorname{curl} \boldsymbol{E}(t) \cdot(\boldsymbol{w}-\boldsymbol{H}(t)) \\
-\boldsymbol{H}(t) \cdot \operatorname{curl}(\boldsymbol{v}-\boldsymbol{E}(t)) d x+\int_{\Omega} \boldsymbol{J}(t) \cdot(\boldsymbol{v}-\boldsymbol{E}(t)) d x=\int_{\Omega} \mathbf{f}(t) \cdot(\boldsymbol{v}-\boldsymbol{E}(t)) d x \\
\text { for a.e. } t \in(0, T) \text { and all }(\boldsymbol{v}, \boldsymbol{w}) \in \boldsymbol{H}_{0}(\operatorname{curl}) \times \boldsymbol{L}^{2}(\Omega) .
\end{array}
$$

On the other hand, in view of $(1.1 \mathrm{~b})$, we have that

$$
\begin{aligned}
\int_{\Omega} \boldsymbol{J}(t) \cdot(\boldsymbol{v}-\boldsymbol{E}(t)) d x & =\int_{\Omega} \boldsymbol{J}(t) \cdot \boldsymbol{v} d x-\int_{\Omega} g|\boldsymbol{E}(t)| d x \\
& \leq \int_{\Omega} g|\boldsymbol{v}| d x-\int_{\Omega} g|\boldsymbol{E}(t)| d x=\varphi(\boldsymbol{v})-\varphi(\boldsymbol{E}(t)) .
\end{aligned}
$$

Applying this inequality to (3.5), we conclude that $(\boldsymbol{E}, \boldsymbol{H}) \in L^{\infty}\left((0, T), \boldsymbol{H}_{0}(\mathbf{c u r l}) \times\right.$ $\boldsymbol{H}(\mathbf{c u r l})) \cap W^{1, \infty}\left((0, T), \boldsymbol{L}_{\epsilon}^{2}(\Omega) \times \boldsymbol{L}_{\mu}^{2}(\Omega)\right)$ satisfies (3.1). This completes the proof.

Corollary 3.2. Let $\mathbf{f} \in W^{1, \infty}\left((0, T), \boldsymbol{L}^{2}(\Omega)\right)$ and $\left(\boldsymbol{E}_{0}, \boldsymbol{H}_{0}\right) \in \boldsymbol{H}_{0}(\mathbf{c u r l}) \times$ $\boldsymbol{H}(\mathbf{c u r l})$ satisfying $\mu \boldsymbol{H}_{0} \in \boldsymbol{H}_{0}(\operatorname{div}=0)$. Then, the unique solution $(\boldsymbol{E}, \boldsymbol{H}) \in L^{\infty}((0, T)$, $\left.\boldsymbol{H}_{0}(\mathbf{c u r l}) \times \boldsymbol{H}(\mathbf{c u r l})\right) \cap W^{1, \infty}\left((0, T), \boldsymbol{L}_{\epsilon}^{2}(\Omega) \times \boldsymbol{L}_{\mu}^{2}(\Omega)\right)$ of the variational inequality (3.1) satisfies

$\mu \frac{d}{d t} \boldsymbol{H}(t) \in \boldsymbol{H}_{0}(\operatorname{div}=0)$ for a.e. $t \in(0, T)$ and $\mu \boldsymbol{H}(t) \in \boldsymbol{H}_{0}(\operatorname{div}=0)$ for all $t \in[0, T]$. 
Proof. Setting $(\boldsymbol{v}, \boldsymbol{w})=(\boldsymbol{E}(t), \boldsymbol{H}(t) \pm \nabla \phi)$ in (3.1) with $\phi \in H^{1}(\Omega)$ yields that

$\int_{\Omega} \mu \frac{d}{d t} \boldsymbol{H}(t) \cdot \nabla \phi d x+\underbrace{\int_{\Omega} \operatorname{curl} \boldsymbol{E}(t) \cdot \nabla \phi d x}_{=0}=0$ for a.e. $t \in(0, T)$ and all $\phi \in H^{1}(\Omega)$,

where we have also used (3.4) and $\operatorname{curl} \nabla \equiv 0$ to deduce that $\int_{\Omega} \operatorname{curl} \boldsymbol{E}(t) \cdot \nabla \phi d x=0$ for a.e. $t \in(0, T)$. Consequently, $\mu \frac{d}{d t} \boldsymbol{H}(t) \in \boldsymbol{H}_{0}(\operatorname{div}=0)$ holds for a.e. $t \in(0, T)$. Furthermore, integrating (3.6) over the time interval $[0, \tau]$, with $\tau \in[0, T]$, implies that

$$
\begin{aligned}
0 & =\int_{\Omega} \mu \boldsymbol{H}(\tau) \cdot \nabla \phi d x-\int_{\Omega} \mu \boldsymbol{H}_{0} \cdot \nabla \phi d x \\
& =\int_{\Omega} \mu \boldsymbol{H}(\tau) \cdot \nabla \phi d x \quad \forall \phi \in H^{1}(\Omega), \quad \forall \tau \in[0, T],
\end{aligned}
$$

since $\mu \boldsymbol{H}_{0} \in \boldsymbol{H}_{0}(\operatorname{div}=0)$. In conclusion, $\mu \boldsymbol{H}(\tau) \in \boldsymbol{H}_{0}(\operatorname{div}=0)$ holds for all $\tau \in[0, T]$. This completes the proof.

We close this section by presenting a variational inequality for (1.1) in the magnetic induction formulation, which is the key basis for our numerical analysis. For the upcoming result, we shall make use of the space

$$
\boldsymbol{R}(\Omega):=\mu \boldsymbol{H}(\text { curl }) \cap \boldsymbol{H}_{0}(\operatorname{div}=0) .
$$

Theorem 3.3. Let $\mathbf{f} \in W^{1, \infty}\left((0, T), \boldsymbol{L}^{2}(\Omega)\right)$ and $\left(\boldsymbol{E}_{0}, \boldsymbol{B}_{0}\right) \in \boldsymbol{H}_{0}(\mathbf{c u r l}) \times \boldsymbol{R}(\Omega)$. Then, the hyperbolic mixed variational inequality

$$
\left\{\begin{array}{l}
\int_{\Omega} \epsilon \frac{d}{d t} \boldsymbol{E}(t) \cdot(\boldsymbol{v}-\boldsymbol{E}(t))+\mu^{-1} \frac{d}{d t} \boldsymbol{B}(t) \cdot(\boldsymbol{w}-\boldsymbol{B}(t)) d x \\
\quad+\int_{\Omega} \mu^{-1}(\operatorname{curl} \boldsymbol{E}(t) \cdot(\boldsymbol{w}-\boldsymbol{B}(t))-\boldsymbol{B}(t) \cdot \operatorname{curl}(\boldsymbol{v}-\boldsymbol{E}(t))) d x \\
\quad+\varphi(\boldsymbol{v})-\varphi(\boldsymbol{E}(t)) \geq \int_{\Omega} \mathbf{f}(t) \cdot(\boldsymbol{v}-\boldsymbol{E}(t)) d x \\
\quad \text { for a.e. } t \in(0, T) \text { and all }(\boldsymbol{v}, \boldsymbol{w}) \in \boldsymbol{H}_{0}(\operatorname{curl}) \times \boldsymbol{L}^{2}(\Omega) \\
\quad(\boldsymbol{E}, \boldsymbol{B})(0)=\left(\boldsymbol{E}_{0}, \boldsymbol{B}_{0}\right)
\end{array}\right.
$$

admits a unique solution $(\boldsymbol{E}, \boldsymbol{B}) \in L^{\infty}\left((0, T), \boldsymbol{H}_{0}(\mathbf{c u r l}) \times \boldsymbol{R}(\Omega)\right) \cap W^{1, \infty}((0, T)$, $\boldsymbol{L}_{\epsilon}^{2}(\Omega) \times \boldsymbol{H}_{0}(\operatorname{div}=0)$ ). The unique solution $\boldsymbol{E}$ of $(\mathrm{VI})$ with $\boldsymbol{H}:=\mu^{-1} \boldsymbol{B}$ is exactly the unique solution of (3.1).

Proof. By assumption, we have that $\boldsymbol{H}_{0}:=\mu^{-1} \boldsymbol{B}_{0} \in \boldsymbol{H}(\mathbf{c u r l})$, and so $\left(\boldsymbol{E}_{0}, \boldsymbol{H}_{0}\right) \in$ $\boldsymbol{H}_{0}(\mathbf{c u r l}) \times \boldsymbol{H}(\mathbf{c u r l})$ such that, according to Theorem 3.1, the variational inequality (3.1) admits a unique solution $(\boldsymbol{E}, \boldsymbol{H}) \in L^{\infty}\left((0, T), \boldsymbol{H}_{0}(\right.$ curl $) \times \boldsymbol{H}($ curl $\left.)\right) \cap$ $W^{1, \infty}\left((0, T), \boldsymbol{L}_{\epsilon}^{2}(\Omega) \times \boldsymbol{L}_{\mu}^{2}(\Omega)\right)$. We set $\boldsymbol{B}:=\mu \boldsymbol{H}$. Since $\mu \boldsymbol{H}_{0}=\boldsymbol{B}_{0} \in \boldsymbol{H}_{0}(\operatorname{div}=0)$, Corollary 3.2 implies that

$$
\boldsymbol{B}=\mu \boldsymbol{H} \in L^{\infty}((0, T), \boldsymbol{R}(\Omega)) \cap W^{1, \infty}\left((0, T), \boldsymbol{H}_{0}(\operatorname{div}=0)\right) .
$$


On the other hand, by virtue of $(3.1),(\boldsymbol{E}, \boldsymbol{B})=(\boldsymbol{E}, \mu \boldsymbol{H})$ satisfies

$$
\begin{gathered}
\int_{\Omega} \epsilon \frac{d}{d t} \boldsymbol{E}(t) \cdot(\boldsymbol{v}-\boldsymbol{E}(t))+\frac{d}{d t} \boldsymbol{B}(t) \cdot\left(\hat{\boldsymbol{w}}-\mu^{-1} \boldsymbol{B}(t)\right) d x+\int_{\Omega} \operatorname{curl} \boldsymbol{E}(t) \cdot\left(\hat{\boldsymbol{w}}-\mu^{-1} \boldsymbol{B}(t)\right) \\
-\mu^{-1} \boldsymbol{B}(t) \cdot \operatorname{curl}(\boldsymbol{v}-\boldsymbol{E}(t)) d x+\varphi(\boldsymbol{v})-\varphi(\boldsymbol{E}(t)) \geq \int_{\Omega} \mathbf{f}(t) \cdot(\boldsymbol{v}-\boldsymbol{E}(t)) d x
\end{gathered}
$$

for a.e. $t \in(0, T)$ and all $(\boldsymbol{v}, \hat{\boldsymbol{w}}) \in \boldsymbol{H}_{0}(\mathbf{c u r l}) \times \boldsymbol{L}^{2}(\Omega)$. Therefore, setting $\hat{\boldsymbol{w}}=\mu^{-1} \boldsymbol{w}$, with $\boldsymbol{w} \in \boldsymbol{L}^{2}(\Omega)$, in (3.7) implies that $(\boldsymbol{E}, \boldsymbol{B})$ is a solution of (VI). On the other hand, by a similar transformation, every solution of (VI) satisfies (3.1) with $\boldsymbol{H}=\mu \boldsymbol{B}$. Therefore, the assertion follows.

4. Semidiscrete Ritz-Galerkin approximation. Let $\boldsymbol{V}_{h} \subset \boldsymbol{H}_{0}(\mathrm{curl})$ and $\boldsymbol{W}_{h} \subset \boldsymbol{L}^{2}(\Omega)$ be two families of finite-dimensional subspaces, depending on parameters $h>0$. They are assumed to satisfy the following two conditions:

(B1) The family $\boldsymbol{V}_{h} \subset \boldsymbol{H}_{0}($ curl $)$ is dense:

$$
\forall \boldsymbol{v} \in \boldsymbol{H}_{0}\left(\text { curl) } \forall \delta>0 \exists \bar{h}>0 \forall h \in(0, \bar{h}) \exists \boldsymbol{v}_{h} \in \boldsymbol{V}_{h}:\left\|\boldsymbol{v}_{h}-\boldsymbol{v}\right\|_{\boldsymbol{H}(\text { curl })} \leq \delta .\right.
$$

(B2) The inclusion curl $\boldsymbol{V}_{h} \subset \boldsymbol{W}_{h}$ is satisfied for all $h>0$.

Let us underline that, in contrast to (B1), the family $\boldsymbol{W}_{h} \subset \boldsymbol{L}^{2}(\Omega)$ is not necessarily dense. From now on, let $\left(\boldsymbol{E}_{0}, \boldsymbol{B}_{0}\right) \in \boldsymbol{H}_{0}(\mathbf{c u r l}) \times \boldsymbol{R}(\Omega)$ and $\mathbf{f} \in W^{1, \infty}\left((0, T), \boldsymbol{L}^{2}(\Omega)\right)$. Then, we formulate the semidiscrete Ritz-Galerkin approximation for (VI) as follows: Given a proper approximation $\left(\boldsymbol{E}_{0 h}, \boldsymbol{B}_{0 h}\right) \in \boldsymbol{V}_{h} \times \boldsymbol{W}_{h}$ of $\left(\boldsymbol{E}_{0}, \boldsymbol{B}_{0}\right)$, find $\left(\boldsymbol{E}_{h}, \boldsymbol{B}_{h}\right) \in$ $H^{1}\left((0, T), \boldsymbol{V}_{h} \times \boldsymbol{W}_{h}\right)$ such that

$$
\left\{\begin{array}{l}
\int_{\Omega} \epsilon \frac{d}{d t} \boldsymbol{E}_{h}(t) \cdot\left(\boldsymbol{v}_{h}-\boldsymbol{E}_{h}(t)\right)+\mu^{-1} \frac{d}{d t} \boldsymbol{B}_{h}(t) \cdot\left(\boldsymbol{w}_{h}-\boldsymbol{B}_{h}(t)\right) d x \\
\quad+\int_{\Omega} \mu^{-1}\left(\operatorname{curl} \boldsymbol{E}_{h}(t) \cdot\left(\boldsymbol{w}_{h}-\boldsymbol{B}_{h}(t)\right)-\boldsymbol{B}_{h}(t) \cdot \operatorname{curl}\left(\boldsymbol{v}_{h}-\boldsymbol{E}_{h}(t)\right)\right) d x \\
\quad+\varphi\left(\boldsymbol{v}_{h}\right)-\varphi\left(\boldsymbol{E}_{h}(t)\right) \geq \int_{\Omega} \mathbf{f}(t) \cdot\left(\boldsymbol{v}_{h}-\boldsymbol{E}_{h}(t)\right) d x \\
\text { for a.e. } t \in(0, T) \text { and all }(\boldsymbol{v}, \boldsymbol{w}) \in \boldsymbol{V}_{h} \times \boldsymbol{W}_{h}, \\
\quad(\boldsymbol{E}, \boldsymbol{B})(0)=\left(\boldsymbol{E}_{0 h}, \boldsymbol{B}_{0 h}\right) .
\end{array}\right.
$$

Let $\left\{\boldsymbol{\psi}_{h}^{j}\right\}_{j=1}^{N_{h}} \subset \boldsymbol{V}_{h}$ and $\left\{\boldsymbol{\eta}_{h}^{j}\right\}_{j=1}^{M_{h}} \subset \boldsymbol{W}_{h}$ denote bases for $\boldsymbol{V}_{h}$ and $\boldsymbol{W}_{h}$, respectively. Then, for every $\left(\boldsymbol{v}_{h}, \boldsymbol{w}_{h}\right) \in \boldsymbol{V}_{h} \times \boldsymbol{W}_{h}$, there exists a unique vector $y=\left(\begin{array}{l}v \\ w\end{array}\right) \in \mathbb{R}^{N_{h}+M_{h}}$ such that

$$
\boldsymbol{v}_{h}=\sum_{j=1}^{N_{h}} v_{j} \boldsymbol{\psi}_{h}^{j} \quad \text { and } \quad \boldsymbol{w}_{h}=\sum_{j=1}^{M_{h}} w_{j} \boldsymbol{\eta}_{h}^{j} .
$$

Making use of the bases, we introduce the matrices

$$
\begin{aligned}
\mathbb{M}_{h}^{(\epsilon)} & :=\left(\int_{\Omega} \epsilon \boldsymbol{\psi}_{h}^{i} \cdot \boldsymbol{\psi}_{h}^{j} d x\right)_{i j} \in \mathbb{R}^{N_{h} \times N_{h}}, \quad \mathbb{M}_{h}^{(\mu)}:=\left(\int_{\Omega} \mu^{-1} \boldsymbol{\eta}_{h}^{i} \cdot \boldsymbol{\eta}_{h}^{j} d x\right)_{i j} \in \mathbb{R}^{M_{h} \times M_{h}}, \\
\mathbb{K}_{h}^{(\mu)} & :=\left(\int_{\Omega} \mu^{-1} \boldsymbol{\eta}_{h}^{i} \cdot \operatorname{curl} \boldsymbol{\psi}_{h}^{j} d x\right)_{i j} \in \mathbb{R}^{M_{h} \times N_{h}},
\end{aligned}
$$


and

$\mathbb{K}_{h}:=\left(\begin{array}{cc}0 & -\mathbb{K}_{h}^{(\mu)^{T}} \\ \mathbb{K}_{h}^{(\mu)} & 0\end{array}\right) \in \mathbb{R}^{N_{h}+M_{h} \times N_{h}+M_{h}}, \mathbb{M}_{h}:=\left(\begin{array}{cc}\mathbb{M}_{h}^{(\epsilon)} & 0 \\ 0 & \mathbb{M}_{h}^{(\mu)}\end{array}\right) \in \mathbb{R}^{N_{h}+M_{h} \times N_{h}+M_{h}}$,

as well as the following function:

$$
\varphi_{h}: \mathbb{R}^{N_{h}+M_{h}} \rightarrow \mathbb{R}, \quad \varphi_{h}(v)=\varphi\left(\sum_{j=1}^{N_{h}} v_{j} \psi_{h}^{j}\right)=\int_{\Omega} g(x)\left|\sum_{j=1}^{N_{h}} v_{j} \boldsymbol{\psi}_{h}^{j}(x)\right| d x .
$$

Now, by the representation through the bases (4.1) and making use of the matrices (4.2) and the function (4.3), we see that $\left(\mathrm{VI}_{h}\right)$ is equivalent to the following evolution variational inequality on $\mathbb{R}^{N_{h}+M_{h}}$ : Find $y \in H^{1}\left((0, T), \mathbb{R}^{N_{h}+M_{h}}\right)$ such that

$$
\left\{\begin{array}{l}
\left(v-y(t), \mathbb{M}_{h} \frac{d}{d t} y(t)+\mathbb{K}_{h} y(t)\right)_{\mathbb{R}^{N_{h}+M_{h}}}+\varphi_{h}(v)-\varphi_{h}(y(t)) \\
\geq(v-y(t), z(t))_{\mathbb{R}^{N_{h}+M_{h}}} \quad \forall v \in \mathbb{R}^{N_{h}+M_{h}} \text { and a.e. } t \in(0, T), \\
y(0)=y_{0},
\end{array}\right.
$$

where $y_{0}=\left(\begin{array}{c}E_{0} \\ B_{0}\end{array}\right) \in \mathbb{R}^{N_{h}+M_{h}}$ is the unique vector satisfying $\boldsymbol{E}_{0 h}=\sum_{j=1}^{N_{h}} E_{0_{j}} \boldsymbol{\psi}_{h}^{j}$ and $\boldsymbol{B}_{0 h}=\sum_{j=1}^{M_{h}} B_{0_{j}} \boldsymbol{\eta}_{h}^{j}$. Moreover, the function $z \in W^{1, \infty}\left((0, T), \mathbb{R}^{N_{h}+M_{h}}\right)$ is defined by $z_{j}(t):=\int_{\Omega} \mathbf{f}(t) \cdot \boldsymbol{\psi}_{h}^{j} d x \quad \forall j \in\left\{1, \ldots, N_{h}\right\}, \quad z_{j}(t):=0, \quad \forall j \in\left\{N_{h}+1, \ldots, N_{h}+M_{h}\right\}$.

By a classical result (see, e.g., [3, Theorem 4.1, p. 124]), (4.4) admits a unique solution $y \in W^{1, \infty}\left((0, T), \mathbb{R}^{N_{h}+M_{h}}\right)$. In all of what follows, for every $h>0$, let $\left(\boldsymbol{E}_{h}, \boldsymbol{B}_{h}\right) \in$ $W^{1, \infty}\left((0, T), \boldsymbol{V}_{h} \times \boldsymbol{W}_{h}\right)$ denote the unique solution of the Ritz-Galerkin approximation $\left(\mathrm{VI}_{h}\right)$, and let $(\boldsymbol{E}, \boldsymbol{B}) \in L^{\infty}\left((0, T), \boldsymbol{H}_{0}(\mathbf{c u r l}) \times \boldsymbol{R}(\Omega)\right) \cap W^{1, \infty}\left((0, T), \boldsymbol{L}_{\epsilon}^{2}(\Omega) \times\right.$ $\boldsymbol{H}_{0}($ div $\left.=0)\right)$ denote the unique solution of $(\mathrm{VI})$. Note that, possibly after a modification on a set of $[0, T]$ with measure zero, they also satisfy

$(\boldsymbol{E}, \boldsymbol{B}) \in \mathcal{C}\left([0, T], \boldsymbol{L}_{\epsilon}^{2}(\Omega) \times \boldsymbol{H}_{0}(\operatorname{div}=0)\right) \quad$ and $\quad\left(\boldsymbol{E}_{h}, \boldsymbol{B}_{h}\right) \in \mathcal{C}\left([0, T], \boldsymbol{V}_{h} \times \boldsymbol{W}_{h}\right)$.

In the upcoming lemmas, we analyze the structural property of the solution to $\left(\mathrm{VI}_{h}\right)$ and its stability. These results will be important for the convergence and error analysis of $\left(\mathrm{VI}_{h}\right)$.

LEMMA 4.1. For a.e. $t \in(0, T)$ and all $h>0$, it holds that

$$
\begin{aligned}
\frac{d}{d t} \boldsymbol{B}_{h}(t) & =-\operatorname{curl} \boldsymbol{E}_{h}(t), \quad \frac{d}{d t} \boldsymbol{B}(t)=-\operatorname{curl} \boldsymbol{E}(t), \\
\int_{\Omega} \epsilon & \left(\frac{d}{d t} \boldsymbol{E}(t)-\frac{d}{d t} \boldsymbol{E}_{h}(t)\right) \cdot \boldsymbol{v}_{h} d x \\
& =\int_{\Omega} \mu^{-1}\left(\boldsymbol{B}(t)-\boldsymbol{B}_{h}(t)\right) \cdot \operatorname{curl} \boldsymbol{v}_{h} d x \quad \forall \boldsymbol{v}_{h} \in \boldsymbol{V}_{h} .
\end{aligned}
$$

Proof. Let $h>0$. Setting $\left(\boldsymbol{v}_{h}, \boldsymbol{w}_{h}\right)=0$ and $\left(\boldsymbol{v}_{h}, \boldsymbol{w}_{h}\right)=\left(2 \boldsymbol{E}_{h}(t), 2 \boldsymbol{B}_{h}(t)\right)$ in $\left(\mathrm{VI}_{h}\right)$ implies

$$
\begin{gathered}
\int_{\Omega} \epsilon \frac{d}{d t} \boldsymbol{E}_{h}(t) \cdot \boldsymbol{E}_{h}(t)+\mu^{-1} \frac{d}{d t} \boldsymbol{B}_{h}(t) \cdot \boldsymbol{B}_{h}(t) d x+\varphi\left(\boldsymbol{E}_{h}(t)\right) \\
=\int_{\Omega} \mathbf{f}(t) \cdot \boldsymbol{E}_{h}(t) d x \text { for a.e. } t \in(0, T) .
\end{gathered}
$$


Then, applying (4.7) to $\left(\mathrm{VI}_{h}\right)$, we see that $\left(\boldsymbol{E}_{h}, \boldsymbol{B}_{h}\right)$ satisfies

$$
\begin{aligned}
& \int_{\Omega} \epsilon \frac{d}{d t} \boldsymbol{E}_{h}(t) \cdot \boldsymbol{v}_{h}+\mu^{-1} \frac{d}{d t} \boldsymbol{B}_{h}(t) \cdot \boldsymbol{w}_{h} d x \\
& \quad+\int_{\Omega} \mu^{-1}\left(\operatorname{curl} \boldsymbol{E}_{h}(t) \cdot \boldsymbol{w}_{h}-\boldsymbol{B}_{h}(t) \cdot \operatorname{curl} \boldsymbol{v}_{h}\right) d x+\varphi\left(\boldsymbol{v}_{h}\right) \\
& \quad \geq \int_{\Omega} \mathbf{f}(t) \cdot \boldsymbol{v}_{h} d x \quad \text { for a.e. } t \in(0, T) \quad \text { and all }\left(\boldsymbol{v}_{h}, \boldsymbol{w}_{h}\right) \in \boldsymbol{V}_{h} \times \boldsymbol{W}_{h} .
\end{aligned}
$$

As $\operatorname{curl} \boldsymbol{V}_{h} \subset \boldsymbol{W}_{h}$, we may now set $\boldsymbol{v}_{h}=0$ and $\boldsymbol{w}_{h}=-\left(\frac{d}{d t} \boldsymbol{B}_{h}(t)+\operatorname{curl} \boldsymbol{E}_{h}(t)\right)$ in (4.8) to get

$$
\int_{\Omega} \mu^{-1}\left|\frac{d}{d t} \boldsymbol{B}_{h}(t)+\operatorname{curl} \boldsymbol{E}_{h}(t)\right|^{2} d x \leq 0 \Longrightarrow \frac{d}{d t} \boldsymbol{B}_{h}(t)=-\operatorname{curl} \boldsymbol{E}_{h}(t) \text { for a.e. } t \in(0, T) .
$$

Using the above identity in (4.8), we obtain

$$
\int_{\Omega} \epsilon \frac{d}{d t} \boldsymbol{E}_{h}(t) \cdot \boldsymbol{v}_{h}-\int_{\Omega} \mu^{-1} \boldsymbol{B}_{h}(t) \cdot \operatorname{curl} \boldsymbol{v}_{h} d x+\varphi\left(\boldsymbol{v}_{h}\right) \geq \int_{\Omega} \mathbf{f}(t) \cdot \boldsymbol{v}_{h} d x
$$

for a.e. $t \in(0, T)$ and all $\boldsymbol{v}_{h} \in \boldsymbol{V}_{h}$.

Analogously, by setting $(\boldsymbol{v}, \boldsymbol{w})=0$ and $(\boldsymbol{v}, \boldsymbol{w})=(2 \boldsymbol{E}(t), 2 \boldsymbol{B}(t))$ in (VI), we infer that the solution $(\boldsymbol{E}, \boldsymbol{B})$ of (VI) satisfies

$$
\begin{aligned}
& \int_{\Omega} \epsilon \frac{d}{d t} \boldsymbol{E}(t) \cdot \boldsymbol{v}+\mu^{-1} \frac{d}{d t} \boldsymbol{B}(t) \cdot \boldsymbol{w} d x+\int_{\Omega} \mu^{-1}(\operatorname{curl} \boldsymbol{E}(t) \cdot \boldsymbol{w}-\boldsymbol{B}(t) \cdot \operatorname{curl} \boldsymbol{v}) d x \\
& \quad+\varphi(\boldsymbol{v}) \geq \int_{\Omega} \mathbf{f}(t) \cdot \boldsymbol{v} d x \quad \text { for a.e. } t \in(0, T) \text { and all }(\boldsymbol{v}, \boldsymbol{w}) \in \boldsymbol{H}_{0}(\operatorname{curl}) \times \boldsymbol{L}^{2}(\Omega) .
\end{aligned}
$$

Setting $\boldsymbol{v}=0$ and $\boldsymbol{w}=-\left(\frac{d}{d t} \boldsymbol{B}(t)+\operatorname{curl} \boldsymbol{E}(t)\right)$ in (4.10), we get

$$
\frac{d}{d t} \boldsymbol{B}(t)=-\operatorname{curl} \boldsymbol{E}(t) \quad \text { for a.e. } t \in(0, T) .
$$

Applying this identity to (4.10), we obtain

$$
\int_{\Omega} \epsilon \frac{d}{d t} \boldsymbol{E}(t) \cdot \boldsymbol{v}-\int_{\Omega} \mu^{-1} \boldsymbol{B}(t) \cdot \operatorname{curl} \boldsymbol{v} d x+\varphi(\boldsymbol{v}) \geq \int_{\Omega} \mathbf{f}(t) \cdot \boldsymbol{v} d x
$$

for a.e. $t \in(0, T)$ and all $\boldsymbol{v} \in \boldsymbol{H}_{0}$ (curl). Finally, subtracting (4.9) from (4.11) with $\boldsymbol{v}=\boldsymbol{v}_{h} \in \boldsymbol{V}_{h}$ implies for a.e. $t \in(0, T)$ and all $h>0$ that

$$
\int_{\Omega} \epsilon\left(\frac{d}{d t} \boldsymbol{E}(t)-\frac{d}{d t} \boldsymbol{E}_{h}(t)\right) \cdot \boldsymbol{v}_{h} d x=\int_{\Omega} \mu^{-1}\left(\boldsymbol{B}(t)-\boldsymbol{B}_{h}(t)\right) \cdot \operatorname{curl} \boldsymbol{v}_{h} d x \quad \forall \boldsymbol{v}_{h} \in \boldsymbol{V}_{h} .
$$

This completes the proof.

Lemma 4.2. For every $h>0$, the solution $\left(\boldsymbol{E}_{h}, \boldsymbol{B}_{h}\right)$ of $\left(\mathrm{VI}_{h}\right)$ satisfies the estimate

$$
\begin{aligned}
\left\|\left(\boldsymbol{E}_{h}, \boldsymbol{B}_{h}\right)\right\|_{\mathcal{C}\left([0, T], \boldsymbol{L}_{\epsilon}^{2}(\Omega) \times \boldsymbol{L}_{1 / \mu}^{2}(\Omega)\right)} \leq & \left\|\left(\boldsymbol{E}_{0 h}, \boldsymbol{B}_{0 h}\right)\right\|_{\boldsymbol{L}_{\epsilon}^{2}(\Omega) \times \boldsymbol{L}_{1 / \mu}^{2}(\Omega)} \\
& +2 \underline{\epsilon}^{-1 / 2}\left(\|\mathbf{f}\|_{L^{1}\left((0, T), \boldsymbol{L}^{2}(\Omega)\right)}+T\|g\|_{L^{2}(\Omega)}\right) .
\end{aligned}
$$

In particular, if $\left\{\left(\boldsymbol{E}_{0 h}, \boldsymbol{B}_{0 h}\right)\right\}_{h>0} \subset \boldsymbol{L}_{\epsilon}^{2}(\Omega) \times \boldsymbol{L}_{1 / \mu}^{2}(\Omega)$ is bounded, then $\left\{\left(\boldsymbol{E}_{h}, \boldsymbol{B}_{h}\right)\right\}_{h>0}$ is bounded in $\mathcal{C}\left([0, T], \boldsymbol{L}_{\epsilon}^{2}(\Omega) \times \boldsymbol{L}_{1 / \mu}^{2}(\Omega)\right)$. 
Proof. Let $h>0$. Integrating (4.7) over the time interval $[0, \tau]$ with $\tau \in[0, T]$ yields

$$
\begin{aligned}
& \frac{1}{2}\left\|\left(\boldsymbol{E}_{h}, \boldsymbol{B}_{h}\right)(\tau)\right\|_{\boldsymbol{L}_{\epsilon}^{2}(\Omega) \times \boldsymbol{L}_{1 / \mu}^{2}(\Omega)}^{2} \\
& \quad=\frac{1}{2}\left\|\left(\boldsymbol{E}_{h}, \boldsymbol{B}_{h}\right)(0)\right\|_{\boldsymbol{L}_{\epsilon}^{2}(\Omega) \times \boldsymbol{L}_{1 / \mu}^{2}(\Omega)}^{2}+\int_{0}^{\tau} \int_{\Omega} \mathbf{f}(t) \cdot \boldsymbol{E}_{h}(t) d x d t+\int_{0}^{\tau} \int_{\Omega} g\left|\boldsymbol{E}_{h}(t)\right| d t \\
& \quad \leq \frac{1}{2}\left\|\left(\boldsymbol{E}_{0 h}, \boldsymbol{B}_{0 h}\right)\right\|_{\boldsymbol{L}_{\epsilon}^{2}(\Omega) \times \boldsymbol{L}_{1 / \mu}^{2}(\Omega)}^{2}+\left\|\boldsymbol{E}_{h}\right\|_{\mathcal{C}\left([0, T], \boldsymbol{L}^{2}(\Omega)\right)}\left(\|\mathbf{f}\|_{L^{1}\left((0, T), \boldsymbol{L}^{2}(\Omega)\right)}+T\|g\|_{L^{2}(\Omega)}\right) \\
& \quad \leq\left\|\left(\boldsymbol{E}_{h}, \boldsymbol{B}_{h}\right)\right\|_{\mathcal{C}\left([0, T], \boldsymbol{L}_{\epsilon}^{2}(\Omega) \times \boldsymbol{L}_{1 / \mu}^{2}(\Omega)\right)} \\
& \quad \times\left(\frac{1}{2}\left\|\left(\boldsymbol{E}_{0 h}, \boldsymbol{B}_{0 h}\right)\right\|_{\boldsymbol{L}_{\epsilon}^{2}(\Omega) \times \boldsymbol{L}_{1 / \mu}^{2}(\Omega)}+\underline{\epsilon}^{-1 / 2}\left(\|\mathbf{f}\|_{L^{1}\left((0, T), \boldsymbol{L}^{2}(\Omega)\right)}+T\|g\|_{L^{2}(\Omega)}\right)\right) .
\end{aligned}
$$

Since the above inequality holds for all $\tau \in[0, T]$, we come to the conclusion that

$$
\begin{aligned}
\frac{1}{2}\left\|\left(\boldsymbol{E}_{h}, \boldsymbol{B}_{h}\right)\right\|_{\mathcal{C}\left([0, T], \boldsymbol{L}_{\epsilon}^{2}(\Omega) \times \boldsymbol{L}_{1 / \mu}^{2}(\Omega)\right) \leq} & \frac{1}{2}\left\|\left(\boldsymbol{E}_{0 h}, \boldsymbol{B}_{0 h}\right)\right\|_{\boldsymbol{L}_{\epsilon}^{2}(\Omega) \times \boldsymbol{L}_{1 / \mu}^{2}(\Omega)} \\
& +\underline{\epsilon}^{-1 / 2}\left(\|\mathbf{f}\|_{L^{1}\left((0, T), \boldsymbol{L}^{2}(\Omega)\right)}+T\|g\|_{L^{2}(\Omega)}\right) .
\end{aligned}
$$

This completes the proof.

4.1. Convergence analysis. Our goal now is to prove the convergence of the solution of $\left(\mathrm{VI}_{h}\right)$ toward the one of (VI) as $h \rightarrow 0$. In all of what follows, we endow the Hilbert space $\boldsymbol{H}_{0}(\operatorname{div}=0)$ with the weighted scalar product $\left(\mu^{-1} \cdot, \cdot\right)_{\boldsymbol{L}^{2}(\Omega)}$, and we define the following subspace:

$$
\left(\operatorname{curl} \boldsymbol{V}_{h}\right)^{\perp}:=\left\{\hat{\boldsymbol{y}} \in \boldsymbol{H}_{0}(\operatorname{div}=0) \mid\left(\mu^{-1} \hat{\boldsymbol{y}}, \operatorname{curl} \boldsymbol{v}_{h}\right)_{\boldsymbol{L}^{2}(\Omega)}=0 \quad \forall \boldsymbol{v}_{h} \in \boldsymbol{V}_{h}\right\} .
$$

As curl $\boldsymbol{V}_{h}$ is a closed subspace of $\boldsymbol{H}_{0}(\operatorname{div}=0)$, the Hilbert projection theorem implies that

$$
\boldsymbol{H}_{0}(\operatorname{div}=0)=\operatorname{curl} \boldsymbol{V}_{h} \oplus\left(\operatorname{curl} \boldsymbol{V}_{h}\right)^{\perp} .
$$

In other words, for every $\boldsymbol{y} \in \boldsymbol{H}_{0}(\operatorname{div}=0)$, there exists a unique pair $\left(\boldsymbol{v}_{h}, \hat{\boldsymbol{y}}\right) \in \boldsymbol{V}_{h} \times$ $\left(\operatorname{curl} \boldsymbol{V}_{h}\right)^{\perp}$ such that $\boldsymbol{y}=\operatorname{curl} \boldsymbol{v}_{h}+\hat{\boldsymbol{y}}$. We denote the Hilbert projection operator associated with (4.12) by

$$
\boldsymbol{\Pi}_{h}: \boldsymbol{H}_{0}(\operatorname{div}=0) \rightarrow \operatorname{curl} \boldsymbol{V}_{h} .
$$

Accordingly, the Hilbert projection operator $\boldsymbol{\Pi}_{h}: \boldsymbol{H}_{0}(\operatorname{div}=0) \rightarrow \operatorname{curl} \boldsymbol{V}_{h}$ satisfies

$$
\left(\mu^{-1}\left(\boldsymbol{\Pi}_{h} \boldsymbol{y}-\boldsymbol{y}\right), \operatorname{curl} \boldsymbol{v}_{h}\right)_{\boldsymbol{L}^{2}(\Omega)}=0 \quad \forall \boldsymbol{y} \in \boldsymbol{H}_{0}(\operatorname{div}=0), \quad \forall \boldsymbol{v}_{h} \in \boldsymbol{V}_{h}
$$

Lemma 4.3. Assume that the domain $\Omega$ is additionally simply connected. Then, the Hilbert projection operator $\boldsymbol{\Pi}_{h}: \boldsymbol{H}_{0}(\operatorname{div}=0) \rightarrow \operatorname{curl} \boldsymbol{V}_{h}$ satisfies

$$
\lim _{h \rightarrow 0}\left\|\boldsymbol{\Pi}_{h} \boldsymbol{y}-\boldsymbol{y}\right\|_{\boldsymbol{L}_{1 / \mu}^{2}(\Omega)}=0 \quad \forall \boldsymbol{y} \in \boldsymbol{H}_{0}(\operatorname{div}=0) .
$$

Proof. Let $\boldsymbol{y} \in \boldsymbol{H}_{0}(\operatorname{div}=0)$. According to definition and (4.13), it holds that

$$
\|\boldsymbol{y}\|_{L_{1 / \mu}^{2}(\Omega)}^{2}=\left\|\boldsymbol{\Pi}_{h} \boldsymbol{y}\right\|_{L_{1 / \mu}^{2}(\Omega)}^{2}+\left\|\boldsymbol{y}-\boldsymbol{\Pi}_{h} \boldsymbol{y}\right\|_{\boldsymbol{L}_{1 / \mu}^{2}(\Omega)}^{2} \quad \forall h>0
$$


from which it follows that $\left\|\boldsymbol{\Pi}_{h} \boldsymbol{y}\right\|_{L_{1 / \mu}^{2}(\Omega)} \leq\|\boldsymbol{y}\|_{\boldsymbol{L}_{1 / \mu}^{2}(\Omega)}$ for all $h>0$. Therefore, we can find a $\widetilde{\boldsymbol{y}} \in \boldsymbol{H}_{0}(\operatorname{div}=0)$ and a null sequence $\left\{h_{n}\right\}_{n=1}^{\infty}$ of positive real numbers such that

$$
\boldsymbol{\Pi}_{h_{n}} \boldsymbol{y} \rightarrow \widetilde{\boldsymbol{y}} \quad \text { weakly in } \boldsymbol{H}_{0}(\operatorname{div}=0) \quad \text { as } n \rightarrow \infty .
$$

Since $\Omega$ is simply connected, it holds that

$$
\boldsymbol{H}_{0}(\operatorname{div}=0)=\operatorname{curl}\left(\boldsymbol{H}_{0}(\operatorname{curl}) \cap \boldsymbol{H}(\operatorname{div}=0)\right) .
$$

See, e.g., [15, Theorem 36(2), p. 48]. Therefore, there exist $\boldsymbol{v}, \widetilde{\boldsymbol{v}} \in \boldsymbol{H}_{0}(\mathbf{c u r l}) \cap$ $\boldsymbol{H}(\operatorname{div}=0)$ such that

$$
\operatorname{curl} \boldsymbol{v}=\boldsymbol{y} \quad \text { and } \quad \operatorname{curl} \widetilde{\boldsymbol{v}}=\widetilde{\boldsymbol{y}} .
$$

Since the family $\boldsymbol{V}_{h} \subset \boldsymbol{H}_{0}$ (curl) is dense (see (B1)), we can find a subsequence of the null sequence $\left\{h_{n}\right\}_{n=1}^{\infty}$, which we denote again by $\left\{h_{n}\right\}_{n=1}^{\infty}$, so that there exists $\left\{\boldsymbol{v}_{h_{n}}\right\}_{n=1}^{\infty}$ satisfying $\boldsymbol{v}_{h_{n}} \in \boldsymbol{V}_{h_{n}}$ for all $n \in \mathbb{N}$, and

$$
\boldsymbol{v}_{h_{n}} \rightarrow \widetilde{\boldsymbol{v}}-\boldsymbol{v} \text { strongly in } \boldsymbol{H}_{0}(\mathbf{c u r l}) \quad \text { as } n \rightarrow \infty .
$$

Next, by virtue of (4.13), it holds for all $n \in \mathbb{N}$ that $\left(\mu^{-1}\left(\boldsymbol{\Pi}_{h_{n}} \boldsymbol{y}-\boldsymbol{y}\right), \operatorname{curl} \boldsymbol{v}_{h_{n}}\right)_{\boldsymbol{L}^{2}(\Omega)}=$ 0 . Then, passing to the limit $n \rightarrow \infty,(4.14)$ and (4.16) yield

$$
\left(\mu^{-1}(\widetilde{\boldsymbol{y}}-\boldsymbol{y}), \operatorname{curl} \widetilde{\boldsymbol{v}}-\operatorname{curl} \boldsymbol{v}\right)_{\boldsymbol{L}^{2}(\Omega)}=0 \underset{(4.15)}{\Longrightarrow} \widetilde{\boldsymbol{y}}=\boldsymbol{y} .
$$

Since the weak limit $\widetilde{\boldsymbol{y}}=\boldsymbol{y}$ is independent of the sequence $\left\{h_{n}\right\}_{n=1}^{\infty}$, a classical argument implies that (4.14) is satisfied for the whole sequence, i.e,

$$
\boldsymbol{\Pi}_{h} \boldsymbol{y} \rightarrow \boldsymbol{y} \quad \text { weakly in } \boldsymbol{H}_{0}(\operatorname{div}=0) \quad \text { as } h \rightarrow 0 .
$$

On the other hand, we have

$\left\|\boldsymbol{\Pi}_{h} \boldsymbol{y}\right\|_{L_{1 / \mu}^{2}(\Omega)}^{2}=\left(\mu^{-1} \boldsymbol{\Pi}_{h} \boldsymbol{y}, \boldsymbol{\Pi}_{h} \boldsymbol{y}\right)_{L^{2}(\Omega)} \underbrace{=}_{(4.13)}\left(\mu^{-1} \boldsymbol{y}, \boldsymbol{\Pi}_{h} \boldsymbol{y}\right)_{L^{2}(\Omega)} \rightarrow\left(\mu^{-1} \boldsymbol{y}, \boldsymbol{y}\right)_{L^{2}(\Omega)}=\|\boldsymbol{y}\|_{L_{1 / \mu}^{2}(\Omega)}^{2}$,

as $h \rightarrow 0$. Now, the assertion follows from (4.17)-(4.18).

Assumption 4.4. For every $h>0$, there exists a linear bounded operator $\boldsymbol{\Phi}_{h}$ : $\boldsymbol{H}_{0}(\mathbf{c u r l}) \rightarrow \boldsymbol{V}_{h}$ satisfying

$$
\begin{aligned}
& \left(\mu^{-1} \operatorname{curl} \Phi_{h} \boldsymbol{y}, \operatorname{curl} \boldsymbol{v}_{h}\right)_{\boldsymbol{L}^{2}(\Omega)} \\
& \quad=\left(\mu^{-1} \operatorname{curl} \boldsymbol{y}, \operatorname{curl} \boldsymbol{v}_{h}\right)_{\boldsymbol{L}^{2}(\Omega)} \quad \forall \boldsymbol{v}_{h} \in \boldsymbol{V}_{h}, \quad \forall \boldsymbol{y} \in \boldsymbol{H}_{0}(\operatorname{curl}),
\end{aligned}
$$

and

$$
\lim _{h \rightarrow 0}\left\|\boldsymbol{\Phi}_{h} \boldsymbol{y}-\boldsymbol{y}\right\|_{\boldsymbol{L}_{\epsilon}^{2}(\Omega)}=0 \quad \forall \boldsymbol{y} \in \boldsymbol{H}_{0}(\operatorname{curl}) .
$$

Furthermore, there exists a constant $\hat{c}>0$, independent of $h$ and $\boldsymbol{y}$, such that

$$
\left\|\boldsymbol{\Phi}_{h} \boldsymbol{y}\right\|_{\boldsymbol{L}_{\epsilon}^{2}(\Omega)} \leq \hat{c}\|\boldsymbol{y}\|_{\boldsymbol{H}(\text { curl })} \quad \forall h>0, \quad \forall \boldsymbol{y} \in \boldsymbol{H}_{0}(\text { curl }) .
$$


Remark 4.5. Assumption 4.4 can be realized through the solution operator of a mixed discrete variational problem, which we will discuss in section 5 .

Lemma 4.6. Under Assumption 4.4, it holds that

$$
\boldsymbol{\Pi}_{h} \frac{d}{d t} \boldsymbol{B}(t)=-\operatorname{curl}\left(\boldsymbol{\Phi}_{h} \boldsymbol{E}(t)\right)
$$

for a.e. $t \in(0, T)$ and all $h>0$.

Proof. Since the solution of (VI) satisfies $\boldsymbol{B}(t) \in \boldsymbol{H}_{0}(\operatorname{div}=0)$ for all $t \in[0, T]$ and $\frac{d}{d t} \boldsymbol{B}(t) \in \boldsymbol{H}_{0}(\operatorname{div}=0)$ for a.e. $t \in(0, T)$, the property (4.13) implies

$\left(\mu^{-1}\left(\boldsymbol{\Pi}_{h} \frac{d}{d t} \boldsymbol{B}(t)-\frac{d}{d t} \boldsymbol{B}(t)\right), \operatorname{curl} \boldsymbol{v}_{h}\right)_{\boldsymbol{L}^{2}(\Omega)}=0 \quad$ for a.e. $t \in(0, T)$ and all $\boldsymbol{v}_{h} \in \boldsymbol{V}_{h}$.

On the other hand, Lemma 4.1 implies

$$
\begin{aligned}
0 & =\left(\mu^{-1}\left(\frac{d}{d t} \boldsymbol{B}(t)+\operatorname{curl} \boldsymbol{E}(t)\right), \operatorname{curl} \boldsymbol{v}_{h}\right)_{\boldsymbol{L}^{2}(\Omega)} \\
\underbrace{}_{(4.19) \&(4.24)} & \left(\mu^{-1}\left(\boldsymbol{\Pi}_{h} \frac{d}{d t} \boldsymbol{B}(t)+\operatorname{curl}\left(\boldsymbol{\Phi}_{h} \boldsymbol{E}(t)\right)\right), \operatorname{curl} \boldsymbol{v}_{h}\right)_{\boldsymbol{L}^{2}(\Omega)}
\end{aligned}
$$

for a.e. $t \in(0, T)$ and all $\boldsymbol{v}_{h} \in \boldsymbol{V}_{h}$.

Consequently, as $\boldsymbol{\Pi}_{h} \frac{d}{d t} \boldsymbol{B}(t)$ and $\operatorname{curl}\left(\boldsymbol{\Phi}_{h} \boldsymbol{E}(t)\right)$ belong to $\operatorname{curl} \boldsymbol{V}_{h}$ for a.e. $t \in(0, T)$, it follows that $\boldsymbol{\Pi}_{h} \frac{d}{d t} \boldsymbol{B}(t)=-\operatorname{curl}\left(\boldsymbol{\Phi}_{h} \boldsymbol{E}(t)\right)$ for a.e. $t \in(0, T)$.

Theorem 4.7. Let Assumption 4.4 be satisfied. Then, the identity

$$
\begin{aligned}
& \left\|\boldsymbol{E}_{h}(\tau)-\boldsymbol{E}(\tau)\right\|_{\boldsymbol{L}_{\epsilon}^{2}(\Omega)}^{2}+\left\|\boldsymbol{\Pi}_{h} \boldsymbol{B}(\tau)-\boldsymbol{B}_{h}(\tau)\right\|_{\boldsymbol{L}_{1 / \mu}^{2}(\Omega)}^{2}=\left\|\boldsymbol{E}_{0 h}-\boldsymbol{E}_{0}\right\|_{\boldsymbol{L}_{\epsilon}^{2}(\Omega)}^{2} \\
& \quad+\left\|\boldsymbol{\Pi}_{h} \boldsymbol{B}_{0}-\boldsymbol{B}_{0 h}\right\|_{\boldsymbol{L}_{1 / \mu}^{2}(\Omega)}^{2}+2 \int_{0}^{\tau} \int_{\Omega} \epsilon\left(\frac{d}{d t} \boldsymbol{E}(t)-\frac{d}{d t} \boldsymbol{E}_{h}(t)\right)\left(\boldsymbol{E}(t)-\boldsymbol{\Phi}_{h} \boldsymbol{E}(t)\right) d x d t
\end{aligned}
$$

holds for all $\tau \in[0, T]$ and all $h>0$. Furthermore, if $\boldsymbol{E} \in W^{1,1}\left((0, T), \boldsymbol{H}_{0}(\mathbf{c u r l})\right)$, then

$$
\begin{aligned}
& \left\|\boldsymbol{\Phi}_{h} \boldsymbol{E}(\tau)-\boldsymbol{E}_{h}(\tau)\right\|_{\boldsymbol{L}_{\epsilon}^{2}(\Omega)}^{2}+\left\|\boldsymbol{\Pi}_{h} \boldsymbol{B}(\tau)-\boldsymbol{B}_{h}(\tau)\right\|_{\boldsymbol{L}_{1 / \mu}^{2}(\Omega)}^{2}=\left\|\boldsymbol{\Phi}_{h} \boldsymbol{E}_{0}-\boldsymbol{E}_{0 h}\right\|_{\boldsymbol{L}_{\epsilon}^{2}(\Omega)}^{2} \\
& \quad+\left\|\boldsymbol{\Pi}_{h} \boldsymbol{B}_{0}-\boldsymbol{B}_{0 h}\right\|_{\boldsymbol{L}_{1 / \mu}^{2}(\Omega)}^{2}+2 \int_{0}^{\tau} \int_{\Omega} \epsilon\left(\frac{d}{d t} \boldsymbol{E}(t)-\boldsymbol{\Phi}_{h} \frac{d}{d t} \boldsymbol{E}(t)\right)\left(\boldsymbol{E}_{h}(t)-\boldsymbol{\Phi}_{h} \boldsymbol{E}(t)\right) d x d t
\end{aligned}
$$

holds for all $\tau \in[0, T]$ and all $h>0$. Note that the assumption $\boldsymbol{E} \in W^{1,1}((0, T)$, $\left.\boldsymbol{H}_{0}(\mathbf{c u r l})\right) \hookrightarrow \mathcal{C}\left([0, T], \boldsymbol{H}_{0}(\mathbf{c u r l})\right)$ implies possibly after a modification on a subset of $[0, T]$ with zero measure that $\boldsymbol{E} \in \mathcal{C}\left([0, T], \boldsymbol{H}_{0}(\mathbf{c u r l})\right)$ such that $\boldsymbol{\Phi}_{h} \boldsymbol{E} \in \mathcal{C}\left([0, T], \boldsymbol{V}_{h}\right)$. 
Proof. By virtue of Lemmas 4.1 and 4.6, it holds for a.e. $t \in(0, T)$ and every $h>0$ that

$$
\begin{aligned}
& \frac{1}{2} \frac{d}{d t}\left\|\boldsymbol{\Pi}_{h} \boldsymbol{B}(t)-\boldsymbol{B}_{h}(t)\right\|_{\boldsymbol{L}_{1 / \mu}^{2}(\Omega)}^{2} \\
& =\int_{\Omega} \mu^{-1}\left(\boldsymbol{\Pi}_{h} \frac{d}{d t} \boldsymbol{B}(t)-\frac{d}{d t} \boldsymbol{B}_{h}(t)\right) \cdot\left(\boldsymbol{\Pi}_{h} \boldsymbol{B}(t)-\boldsymbol{B}_{h}(t)\right) d x \\
& \underbrace{=}_{(4.5) \&(4.22)} \int_{\Omega} \mu^{-1} \operatorname{curl}\left(\boldsymbol{E}_{h}(t)-\boldsymbol{\Phi}_{h} \boldsymbol{E}(t)\right) \cdot\left(\boldsymbol{\Pi}_{h} \boldsymbol{B}(t)-\boldsymbol{B}_{h}(t)\right) d x \\
& \underbrace{=}_{(4.13)} \int_{\Omega} \mu^{-1} \operatorname{curl}\left(\boldsymbol{E}_{h}(t)-\boldsymbol{\Phi}_{h} \boldsymbol{E}(t)\right) \cdot\left(\boldsymbol{B}(t)-\boldsymbol{B}_{h}(t)\right) d x \\
& \underbrace{=}_{(4.6)} \int_{\Omega} \epsilon\left(\frac{d}{d t} \boldsymbol{E}(t)-\frac{d}{d t} \boldsymbol{E}_{h}(t)\right) \cdot\left(\boldsymbol{E}_{h}(t)-\boldsymbol{\Phi}_{h} \boldsymbol{E}(t)\right) d x,
\end{aligned}
$$

from which it follows that

$$
\begin{gathered}
\frac{1}{2} \frac{d}{d t}\left\|\boldsymbol{E}_{h}(t)-\boldsymbol{E}(t)\right\|_{\boldsymbol{L}_{\epsilon}^{2}(\Omega)}^{2}+\frac{1}{2} \frac{d}{d t}\left\|\boldsymbol{\Pi}_{h} \boldsymbol{B}(t)-\boldsymbol{B}_{h}(t)\right\|_{\boldsymbol{L}_{1 / \mu}^{2}(\Omega)}^{2} \\
\quad=\int_{\Omega} \epsilon\left(\frac{d}{d t} \boldsymbol{E}(t)-\frac{d}{d t} \boldsymbol{E}_{h}(t)\right) \cdot\left(\boldsymbol{E}(t)-\boldsymbol{\Phi}_{h} \boldsymbol{E}(t)\right) d x .
\end{gathered}
$$

Integrating the above identity over the time interval $[0, \tau]$ with $\tau \in[0, T]$ gives (4.25).

Now, suppose that $\boldsymbol{E} \in W^{1,1}\left((0, T), \boldsymbol{H}_{0}(\mathbf{c u r l})\right)$, which implies for every $h>0$ that

$$
\boldsymbol{\Phi}_{h} \boldsymbol{E} \in W^{1,1}\left((0, T), \boldsymbol{L}_{\epsilon}^{2}(\Omega)\right) \quad \text { with } \quad \frac{d}{d t} \boldsymbol{\Phi}_{h} \boldsymbol{E}=\boldsymbol{\Phi}_{h} \frac{d}{d t} \boldsymbol{E} .
$$

Then, in view of (4.28), we obtain from (4.27) that

$$
\begin{aligned}
\frac{1}{2} \frac{d}{d t} & \left\|\boldsymbol{\Pi}_{h} \boldsymbol{B}(t)-\boldsymbol{B}_{h}(t)\right\|_{\boldsymbol{L}_{1 / \mu}^{2}(\Omega)}^{2}=\int_{\Omega} \epsilon\left(\frac{d}{d t} \boldsymbol{\Phi}_{h} \boldsymbol{E}(t)-\frac{d}{d t} \boldsymbol{E}_{h}(t)\right) \cdot\left(\boldsymbol{E}_{h}(t)-\boldsymbol{\Phi}_{h} \boldsymbol{E}(t)\right) d x \\
& +\int_{\Omega} \epsilon\left(\frac{d}{d t} \boldsymbol{E}(t)-\boldsymbol{\Phi}_{h} \frac{d}{d t} \boldsymbol{E}(t)\right) \cdot\left(\boldsymbol{E}_{h}(t)-\boldsymbol{\Phi}_{h} \boldsymbol{E}(t)\right) d x \\
= & -\frac{1}{2} \frac{d}{d t}\left\|\boldsymbol{\Phi}_{h} \boldsymbol{E}(t)-\boldsymbol{E}_{h}(t)\right\|_{\boldsymbol{L}_{\epsilon}^{2}(\Omega)}^{2}+\int_{\Omega} \epsilon\left(\frac{d}{d t} \boldsymbol{E}(t)-\boldsymbol{\Phi}_{h} \frac{d}{d t} \boldsymbol{E}(t)\right) \cdot\left(\boldsymbol{E}_{h}(t)-\boldsymbol{\Phi}_{h} \boldsymbol{E}(t)\right) d x
\end{aligned}
$$

for a.e. $t \in(0, T)$, where for the last equality we have used the following result (see Appendix A): For every $\boldsymbol{u} \in W^{1,1}\left((0, T), \boldsymbol{L}_{\epsilon}^{2}(\Omega)\right)$, the mapping $t \mapsto\|\boldsymbol{u}(t)\|_{\boldsymbol{L}_{\epsilon}^{2}(\Omega)}^{2}$ is of class $W^{1,1}(0, T)$ and

$$
\frac{d}{d t}\|\boldsymbol{u}(t)\|_{\boldsymbol{L}_{\epsilon}^{2}(\Omega)}^{2}=2 \int_{\Omega} \epsilon \boldsymbol{u}^{\prime}(t) \cdot \boldsymbol{u}(t) d x \quad \text { for a.e. } t \in(0, T) .
$$

Integrating (4.29) over the time interval $[0, \tau]$ with $\tau \in[0, T]$ yields finally (4.26).

With Lemmas 4.2 and 4.3 and Theorem 4.7 at hand, we are able to prove a strong convergence result for the Ritz-Galerkin approximation $\left(\mathrm{VI}_{h}\right)$. 
TheOrem 4.8. Assume that $\Omega$ is additionally simply connected and let Assumption 4.4 be satisfied. Furthermore, suppose that

$$
\lim _{h \rightarrow 0}\left\|\boldsymbol{E}_{0 h}-\boldsymbol{E}_{0}\right\|_{\boldsymbol{L}_{\epsilon}^{2}(\Omega)}=0 \quad \text { and } \quad \lim _{h \rightarrow 0}\left\|\boldsymbol{B}_{0 h}-\boldsymbol{B}_{0}\right\|_{\boldsymbol{L}_{1 / \mu}^{2}(\Omega)}=0 .
$$

If $\boldsymbol{E} \in W^{1,1}\left((0, T), \boldsymbol{H}_{0}(\mathbf{c u r l})\right)$ holds or $\left\{\frac{d}{d t} \boldsymbol{E}_{h}\right\}_{h>0}$ is bounded in $L^{1+\theta}\left((0, T), \boldsymbol{L}_{\epsilon}^{2}(\Omega)\right)$ for some $\theta>0$, then

$$
\lim _{h \rightarrow 0}\left\|\boldsymbol{E}_{h}(t)-\boldsymbol{E}(t)\right\|_{\boldsymbol{L}_{\epsilon}^{2}(\Omega)}=0 \quad \text { and } \quad \lim _{h \rightarrow 0}\left\|\boldsymbol{B}_{h}(t)-\boldsymbol{B}(t)\right\|_{\boldsymbol{L}_{1 / \mu}^{2}(\Omega)}=0
$$

hold for all $t \in[0, T]$.

Proof. Case 1. Suppose that $\boldsymbol{E} \in W^{1,1}\left((0, T), \boldsymbol{H}_{0}(\mathbf{c u r l})\right) \hookrightarrow \mathcal{C}\left([0, T], \boldsymbol{H}_{0}(\mathbf{c u r l})\right)$. Then, the convergence property (4.20) implies the following pointwise almost everywhere convergence:

$$
\boldsymbol{\Phi}_{h} \frac{d}{d t} \boldsymbol{E}(t) \rightarrow \frac{d}{d t} \boldsymbol{E}(t) \quad \text { strongly in } \boldsymbol{L}_{\epsilon}^{2}(\Omega) \text { as } h \rightarrow 0 \text { for a.e. } t \in(0, T) .
$$

Moreover, (4.21) yields that

$$
\left\|\boldsymbol{\Phi}_{h} \frac{d}{d t} \boldsymbol{E}(t)\right\|_{\boldsymbol{L}_{\epsilon}^{2}(\Omega)} \leq \hat{c}\left\|\frac{d}{d t} \boldsymbol{E}(t)\right\|_{\boldsymbol{H}(\text { curl })} \quad \text { for a.e. } t \in(0, T) \text { and all } h>0 .
$$

Consequently, as $\frac{d}{d t} \boldsymbol{E} \in L^{1}\left((0, T), \boldsymbol{H}_{0}(\mathbf{c u r l})\right)$, Lebesgue's dominated convergence theorem implies

$$
\lim _{h \rightarrow 0}\left\|\boldsymbol{\Phi}_{h} \frac{d}{d t} \boldsymbol{E}-\frac{d}{d t} \boldsymbol{E}\right\|_{L^{1}\left((0, T), \boldsymbol{L}_{\epsilon}^{2}(\Omega)\right)}=0 .
$$

Moreover, Lemma 4.2 along with (4.21) and $\boldsymbol{E} \in \mathcal{C}\left([0, T], \boldsymbol{H}_{0}(\mathbf{c u r l})\right)$ implies that $\left\{\boldsymbol{E}_{h}-\boldsymbol{\Phi}_{h} \boldsymbol{E}\right\}_{h>0}$ is bounded in $\mathcal{C}\left([0, T], \boldsymbol{L}_{\epsilon}^{2}(\Omega)\right)$. For this reason, we obtain from the above convergence that

$$
\lim _{h \rightarrow 0} \int_{0}^{\tau} \int_{\Omega} \epsilon\left(\frac{d}{d t} \boldsymbol{E}(t)-\boldsymbol{\Phi}_{h} \frac{d}{d t} \boldsymbol{E}(t)\right) \cdot\left(\boldsymbol{E}_{h}(t)-\boldsymbol{\Phi}_{h} \boldsymbol{E}(t)\right) d x d t=0 \quad \forall \tau \in[0, T] .
$$

Applying Lemma 4.3, (4.20), (4.30), and (4.31) to (4.26), we obtain the convergence

$$
\lim _{h \rightarrow 0}\left\|\boldsymbol{\Phi}_{h} \boldsymbol{E}(t)-\boldsymbol{E}_{h}(t)\right\|_{\boldsymbol{L}_{\epsilon}^{2}(\Omega)}=\lim _{h \rightarrow 0}\left\|\boldsymbol{\Pi}_{h} \boldsymbol{B}(t)-\boldsymbol{B}_{h}(t)\right\|_{\boldsymbol{L}_{1 / \mu}^{2}(\Omega)}=0 \quad \forall t \in[0, T] .
$$

On the other hand, as $\boldsymbol{B} \in \mathcal{C}\left([0, T], \boldsymbol{H}_{0}(\operatorname{div}=0)\right)$ and $\boldsymbol{E} \in \mathcal{C}\left([0, T], \boldsymbol{H}_{0}(\mathbf{c u r l})\right)$, Lemma 4.3 and (4.20) yield

$$
\lim _{h \rightarrow 0}\left\|\boldsymbol{\Phi}_{h} \boldsymbol{E}(t)-\boldsymbol{E}(t)\right\|_{\boldsymbol{L}_{\epsilon}^{2}(\Omega)}=\lim _{h \rightarrow 0}\left\|\boldsymbol{\Pi}_{h} \boldsymbol{B}(t)-\boldsymbol{B}(t)\right\|_{\boldsymbol{L}_{1 / \mu}^{2}(\Omega)}=0 \quad \forall t \in[0, T] .
$$

Thus, (4.32) and (4.33) imply the desired convergence.

Case 2. Suppose that $\left\{\frac{d}{d t} \boldsymbol{E}_{h}\right\}_{h>0}$ is bounded in $L^{1+\theta}\left((0, T), \boldsymbol{L}_{\epsilon}^{2}(\Omega)\right)$ for some $\theta>0$. Thanks to (4.20)-(4.21) and $\boldsymbol{E} \in L^{\infty}\left((0, T), \boldsymbol{H}_{0}(\mathbf{c u r l})\right)$, Lebesgue's dominated convergence theorem implies

$$
\lim _{h \rightarrow 0}\left\|\boldsymbol{\Phi}_{h} \boldsymbol{E}-\boldsymbol{E}\right\|_{L^{p}\left((0, T), \boldsymbol{L}_{\epsilon}^{2}(\Omega)\right)}=0 \quad \forall p \in[1, \infty) .
$$


Then, since $\left\{\frac{d}{d t} \boldsymbol{E}_{h}\right\}_{h>0}$ is bounded in $L^{1+\theta}\left((0, T), \boldsymbol{L}_{\epsilon}^{2}(\Omega)\right)$, applying Lemma 4.3, (4.30), and (4.34) to (4.25) yields the convergence

$$
\lim _{h \rightarrow 0}\left\|\boldsymbol{E}_{h}(t)-\boldsymbol{E}(t)\right\|_{\boldsymbol{L}_{\epsilon}^{2}(\Omega)}=\lim _{h \rightarrow 0}\left\|\boldsymbol{\Pi}_{h} \boldsymbol{B}(t)-\boldsymbol{B}_{h}(t)\right\|_{\boldsymbol{L}_{1 / \mu}^{2}(\Omega)}=0 \quad \forall t \in[0, T] .
$$

On the other hand, since $\boldsymbol{B} \in \mathcal{C}\left([0, T], \boldsymbol{H}_{0}(\operatorname{div}=0)\right)$, Lemma 4.3 also implies that

$$
\lim _{h \rightarrow 0}\left\|\boldsymbol{\Pi}_{h} \boldsymbol{B}(t)-\boldsymbol{B}(t)\right\|_{\boldsymbol{L}_{1 / \mu}^{2}(\Omega)}=0 \quad \forall t \in[0, T] .
$$

Concluding from (4.35) and (4.36), we obtain the desired convergence.

5. Mixed finite element method. Throughout this section, $\Omega$ is assumed to be a simply connected Lipschitz polyhedral domain with a connected boundary $\partial \Omega$. We consider a family $\left\{\mathcal{T}_{h}\right\}_{h>0}$ of simplicial triangulations $\mathcal{T}_{h}=\{T\}$ consisting of tetrahedra $T$ such that

$$
\bar{\Omega}=\bigcup_{T \in \mathcal{T}_{h}} T .
$$

For each element $T \in \mathcal{T}_{h}, h_{T}$ denotes the diameter of $T$, and $\rho_{T}$ stands for the diameter of the largest ball contained in $T$. The maximal diameter of all elements is denoted by $h$, i.e., $h:=\max \left\{h_{T} \mid T \in \mathcal{T}_{h}\right\}$. Finally, we suppose that there exist two positive constants $\varrho$ and $\vartheta$ such that

$$
\frac{h_{T}}{\rho_{T}} \leq \varrho \quad \text { and } \quad \frac{h}{h_{T}} \leq \vartheta
$$

hold for all elements $T \in \mathcal{T}_{h}$ and all $h>0$. We choose the space of lowest order edge elements of Nédélec's first family [24] for the finite-dimensional subspace $\boldsymbol{V}_{h} \subset$ $\boldsymbol{H}_{0}(\mathbf{c u r l})$; i.e., we set

$$
\boldsymbol{V}_{h}=\left\{\boldsymbol{v}_{h} \in \boldsymbol{H}_{0}(\text { curl }) \mid \boldsymbol{v}_{h \mid T}=\boldsymbol{a}_{T}+\boldsymbol{b}_{T} \times x \text { with } \boldsymbol{a}_{T}, \boldsymbol{b}_{T} \in \mathbb{R}^{3}, \forall T \in \mathcal{T}_{h}\right\} .
$$

On the other hand, there are three possibilities for the choice of the subspace $\boldsymbol{W}_{h}$ :

$$
\begin{aligned}
& \boldsymbol{W}_{h}=\left\{\boldsymbol{w}_{h} \in \boldsymbol{L}^{2}(\Omega) \mid \boldsymbol{w}_{h \mid T}=\boldsymbol{a}_{T} \text { with } \boldsymbol{a}_{T} \in \mathbb{R}^{3}, \forall T \in \mathcal{T}_{h}\right\}, \\
& \boldsymbol{W}_{h}=\left\{\boldsymbol{w}_{h} \in \boldsymbol{H}_{0}(\operatorname{div}) \mid \boldsymbol{w}_{h \mid T}=\boldsymbol{a}_{T}+\boldsymbol{b}_{T} \cdot x \text { with } \boldsymbol{a}_{T}, \boldsymbol{b}_{T} \in \mathbb{R}^{3}, \forall T \in \mathcal{T}_{h}\right\}, \\
& \boldsymbol{W}_{h}=\operatorname{curl} \boldsymbol{V}_{h} .
\end{aligned}
$$

We remark that (5.2a) is the space of piecewise constant elements. Equation (5.2b) is the space of Raviart-Thomas face elements (cf. [15]), whereas (5.2c) is the divergencefree Raviart-Thomas finite element space. Note that $\operatorname{curl} \boldsymbol{V}_{h}$ contains all piecewise constant divergence-free elements, which is a closed subspace of the Raviart-Thomas finite element space (see [23, p. 150]). For this reason, the choices for $\boldsymbol{W}_{h}$ and $\boldsymbol{V}_{h}$ satisfy the assumptions (B1)-(B2) (see p. 2451). We note that, in the context of linear time-domain Maxwell's equations, the use of the finite element spaces $\boldsymbol{V}_{h}$ and (5.2b) for $\boldsymbol{W}_{h}$ was proposed in [22]. Let us now construct $\boldsymbol{\Phi}_{h}: \boldsymbol{H}_{0}$ (curl) $\rightarrow \boldsymbol{V}_{h}$ from Assumption 4.4 (see p. 2455). To this aim, we introduce the bilinear forms

$$
\begin{array}{lll}
a: \boldsymbol{H}_{0}(\operatorname{curl}) \times \boldsymbol{H}_{0}(\operatorname{curl}) \rightarrow \mathbb{R}, & a(\boldsymbol{y}, \boldsymbol{v}):=\left(\mu^{-1} \operatorname{curl} \boldsymbol{y}, \operatorname{curl} \boldsymbol{v}\right)_{\boldsymbol{L}^{2}(\Omega)}, \\
b: \boldsymbol{H}_{0}(\operatorname{curl}) \times H_{0}^{1}(\Omega) \rightarrow \mathbb{R}, & b(\boldsymbol{y}, \psi):=(\boldsymbol{y}, \nabla \psi)_{\boldsymbol{L}^{2}(\Omega)},
\end{array}
$$


and let $\Theta_{h}$ denote the space of piecewise linear elements with vanishing traces:

$$
\Theta_{h}:=\left\{\phi_{h} \in H_{0}^{1}(\Omega) \mid \phi_{h \mid T}=\boldsymbol{a}_{T} \cdot x+b_{T} \text { with } \boldsymbol{a}_{T} \in \mathbb{R}^{3}, b_{T} \in \mathbb{R}, \forall T \in \mathcal{T}_{h}\right\} .
$$

We define the linear and bounded operator $\boldsymbol{\Phi}_{h}: \boldsymbol{H}_{0}(\mathbf{c u r l}) \rightarrow \boldsymbol{V}_{h}$ as follows: For every $\boldsymbol{y} \in \boldsymbol{H}_{0}(\mathbf{c u r l})$, let $\boldsymbol{\Phi}_{h} \boldsymbol{y}:=\boldsymbol{y}_{h}$ denote the unique solution of the discrete variational mixed problem

$$
\begin{cases}a\left(\boldsymbol{y}_{h}, \boldsymbol{v}_{h}\right)=a\left(\boldsymbol{y}, \boldsymbol{v}_{h}\right) & \forall \boldsymbol{v}_{h} \in \boldsymbol{V}_{h}, \\ b\left(\boldsymbol{y}_{h}, \psi_{h}\right)=b\left(\boldsymbol{y}, \psi_{h}\right) & \forall \psi_{h} \in \Theta_{h} .\end{cases}
$$

Note that the theory of mixed problems (see [23, Theorem 2.45]; cf. [17]) implies that (5.3) admits a unique solution $\boldsymbol{y}_{h}=\boldsymbol{\Phi}_{h} \boldsymbol{y} \in \boldsymbol{V}_{h}$ satisfying

$$
\left\|\boldsymbol{\Phi}_{h} \boldsymbol{y}-\boldsymbol{y}\right\|_{\boldsymbol{H}(\text { curl })} \leq c\left(\inf _{\boldsymbol{\chi}_{h} \in \boldsymbol{V}_{h}}\left\|\boldsymbol{y}-\boldsymbol{\chi}_{h}\right\|_{\boldsymbol{H}(\text { curl })}\right) \quad \forall h>0
$$

with a constant $c>0$, independent of $h$ and $\boldsymbol{y}$. In particular, (5.4) yields

$$
\begin{aligned}
& \lim _{h \rightarrow 0}\left\|\boldsymbol{\Phi}_{h} \boldsymbol{y}-\boldsymbol{y}\right\|_{\boldsymbol{H}(\text { curl })}=0 \quad \forall \boldsymbol{y} \in \boldsymbol{H}_{0}(\text { curl }) \\
& \left\|\boldsymbol{\Phi}_{h} \boldsymbol{y}\right\|_{\boldsymbol{H}(\text { curl })} \leq(c+1)\|\boldsymbol{y}\|_{\boldsymbol{H}(\text { curl })} \quad \forall h>0, \quad \forall \boldsymbol{y} \in \boldsymbol{H}_{0}(\text { curl }) .
\end{aligned}
$$

In conclusion, Assumption 4.4 is satisfied for $\boldsymbol{\Phi}_{h}: \boldsymbol{H}_{0}(\mathbf{c u r l}) \rightarrow \boldsymbol{V}_{h}$, and so our convergence result (Theorem 4.8) is applicable for (5.1)-(5.2).

5.1. Error estimates. We close this paper by proving error estimates for the proposed mixed finite element method. First, we recall classical error estimates for the curl-conforming Nédélec interpolant $\mathcal{N}_{h}$ and the divergence-conforming RaviartThomas interpolant $\boldsymbol{\pi}_{h}$ (see [23, section 5]).

Lemma 5.1 (Theorem 5.25, Theorem 5.41, and Remark 5.42 in [23]). Let $s \in$ $(1 / 2,1]$. There exists a constant $c>0$, independent of $h$ and $\boldsymbol{y}$, such that

$$
\begin{array}{rlrl}
\left\|\boldsymbol{y}-\mathcal{N}_{h} \boldsymbol{y}\right\|_{\boldsymbol{H}(\text { curl })} & \leq c h^{s}\|\boldsymbol{y}\|_{\boldsymbol{H}_{0}^{s}(\text { curl })} & \forall \boldsymbol{y} \in \boldsymbol{H}_{0}^{s}(\mathbf{c u r l}), \quad \forall h>0 \\
\left\|\boldsymbol{y}-\boldsymbol{\pi}_{h} \boldsymbol{y}\right\|_{\boldsymbol{L}^{2}(\Omega)} \leq c h^{s}\|\boldsymbol{y}\|_{\boldsymbol{H}^{s}(\Omega)} & \forall \boldsymbol{y} \in \boldsymbol{H}^{s}(\Omega), \quad \forall h>0
\end{array}
$$

where $\boldsymbol{H}_{0}^{s}(\operatorname{curl}):=\left\{\boldsymbol{y} \in \boldsymbol{H}^{s}(\Omega) \cap \boldsymbol{H}_{0}(\operatorname{curl}) \mid \operatorname{curl} \boldsymbol{y} \in \boldsymbol{H}^{s}(\Omega)\right\}$.

The following auxiliary result is also well known and follows from the above lemma and the embedding result $\boldsymbol{H}_{0}(\mathbf{c u r l}) \cap \boldsymbol{H}(\operatorname{div}=0) \hookrightarrow \boldsymbol{H}^{\xi}(\Omega)$ for some $\xi>\frac{1}{2}$ (see $[2$, Proposition 3.7]).

Lemma 5.2. Let $s \in(1 / 2,1]$. There exists a constant $c>0$, independent of $h$ and $\boldsymbol{y}$, such that

$$
\left\|\boldsymbol{y}-\boldsymbol{\Pi}_{h} \boldsymbol{y}\right\|_{L_{1 / \mu}^{2}(\Omega)} \leq c h^{s}\|\boldsymbol{y}\|_{\boldsymbol{H}^{s}(\Omega)}
$$

for all $\boldsymbol{y} \in \boldsymbol{H}_{0}(\operatorname{div}=0) \cap \boldsymbol{H}^{s}(\Omega)$ and all $h>0$.

Now, we have all the ingredients at hand to prove an a priori error estimate for (5.1)-(5.2).

Assumption 5.3. There exists an $s \in(1 / 2,1]$ such that

$$
\boldsymbol{E}_{0} \in \boldsymbol{H}_{0}^{s}(\operatorname{curl}), \quad \boldsymbol{B}_{0} \in \boldsymbol{R}(\Omega) \cap \boldsymbol{H}^{s}(\Omega),
$$


and the solution $(\boldsymbol{E}, \boldsymbol{B})$ of $(\mathrm{VI})$ satisfies the following additional regularity property:

$$
\left\{\begin{array}{l}
\boldsymbol{E} \in H^{1}\left((0, T), \boldsymbol{H}_{0}^{s}(\mathbf{c u r l})\right) \\
\boldsymbol{B}(t) \in \boldsymbol{H}^{s}(\Omega) \quad \forall t \in[0, T] .
\end{array}\right.
$$

TheOrem 5.4. Let $\boldsymbol{V}_{h}$ be as in (5.1) and $\boldsymbol{W}_{h}$ be given by either (5.2a), (5.2b), or (5.2c). Suppose that Assumption 5.3 is satisfied with $s \in(1 / 2,1]$, and there is a constant $c>0$, independent of $h$, such that

$$
\left\|\boldsymbol{E}_{0 h}-\boldsymbol{E}_{0}\right\|_{\boldsymbol{L}_{\epsilon}^{2}(\Omega)}+\left\|\boldsymbol{B}_{0 h}-\boldsymbol{B}_{0}\right\|_{\boldsymbol{L}_{1 / \mu}^{2}(\Omega)} \leq c h^{s} \quad \forall h>0 .
$$

Then, there exists a constant $c>0$, independent of $h$ and $t$, such that

$$
\begin{gathered}
\left\|\boldsymbol{E}_{h}(t)-\boldsymbol{E}(t)\right\|_{\boldsymbol{L}_{\epsilon}^{2}(\Omega)}+\left\|\boldsymbol{B}_{h}(t)-\boldsymbol{B}(t)\right\|_{\boldsymbol{L}_{1 / \mu}^{2}(\Omega)} \\
\leq c h^{s}\left(\|\boldsymbol{E}(t)\|_{\boldsymbol{H}_{0}^{s}(\mathbf{c u r l})}+\|\boldsymbol{B}(t)\|_{\boldsymbol{H}^{s}(\Omega)}+1\right)
\end{gathered}
$$

for all $h>0$ and all $t \in[0, T]$.

Proof. First, Lemma 5.2, along with $\boldsymbol{B}_{0} \in \boldsymbol{H}^{s}(\Omega)$ and (5.7), implies

$$
\left\|\boldsymbol{\Pi}_{h} \boldsymbol{B}_{0}-\boldsymbol{B}_{0 h}\right\|_{\boldsymbol{L}_{1 / \mu}^{2}(\Omega)} \leq\left\|\boldsymbol{\Pi}_{h} \boldsymbol{B}_{0}-\boldsymbol{B}_{0}\right\|_{\boldsymbol{L}_{1 / \mu}^{2}(\Omega)}+\left\|\boldsymbol{B}_{0}-\boldsymbol{B}_{0 h}\right\|_{\boldsymbol{L}_{1 / \mu}^{2}(\Omega)} \leq c h^{s},
$$

with a constant $c>0$, independent of $h>0$. Furthermore, (5.4)-(5.5), along with $\boldsymbol{E}_{0} \in \boldsymbol{H}_{0}^{s}(\mathbf{c u r l})$ and (5.7), yield that

$$
\left\|\boldsymbol{\Phi}_{h} \boldsymbol{E}_{0}-\boldsymbol{E}_{0 h}\right\|_{\boldsymbol{L}_{\epsilon}^{2}(\Omega)} \leq\left\|\boldsymbol{\Phi}_{h} \boldsymbol{E}_{0}-\boldsymbol{E}_{0}\right\|_{\boldsymbol{L}_{\epsilon}^{2}(\Omega)}+\left\|\boldsymbol{E}_{0}-\boldsymbol{E}_{0 h}\right\|_{\boldsymbol{L}_{\epsilon}^{2}(\Omega)} \leq c h^{s},
$$

with a constant $c>0$, independent of $h>0$. Applying now (5.8)-(5.9) and (5.4)-(5.5) to $(4.26)$, we infer that

$$
\begin{aligned}
& \left\|\boldsymbol{\Phi}_{h} \boldsymbol{E}(t)-\boldsymbol{E}_{h}(t)\right\|_{\boldsymbol{L}_{\epsilon}^{2}(\Omega)}^{2}+\left\|\boldsymbol{\Pi}_{h} \boldsymbol{B}(t)-\boldsymbol{B}_{h}(t)\right\|_{\boldsymbol{L}_{1 / \mu}^{2}(\Omega)}^{2} \\
& \leq c h^{2 s}+2 \int_{0}^{t}\left\|\boldsymbol{\Phi}_{h} \frac{d}{d t} \boldsymbol{E}(\sigma)-\frac{d}{d t} \boldsymbol{E}(\sigma)\right\|_{\boldsymbol{L}_{\epsilon}^{2}(\Omega)}\left\|\boldsymbol{\Phi}_{h} \boldsymbol{E}(\sigma)-\boldsymbol{E}_{h}(\sigma)\right\|_{\boldsymbol{L}_{\epsilon}^{2}(\Omega)} d \sigma \\
& \leq c h^{2 s}+2 \int_{0}^{t} c h^{s}\left\|\frac{d}{d t} \boldsymbol{E}(\sigma)\right\|_{\boldsymbol{H}_{0}^{s}(\mathbf{c u r l})}\left\|\boldsymbol{\Phi}_{h} \boldsymbol{E}(\sigma)-\boldsymbol{E}_{h}(\sigma)\right\|_{\boldsymbol{L}_{\epsilon}^{2}(\Omega)} d \sigma \\
& \leq c h^{2 s}\left(1+\left\|\frac{d}{d t} \boldsymbol{E}(\sigma)\right\|_{L^{2}\left((0, t), \boldsymbol{H}_{0}^{s}(\mathbf{c u r l})\right)}^{2}\right) \\
& \quad+\int_{0}^{t}\left\|\boldsymbol{\Phi}_{h} \boldsymbol{E}(\sigma)-\boldsymbol{E}_{h}(\sigma)\right\|_{\boldsymbol{L}_{\epsilon}^{2}(\Omega)}^{2} d \sigma \quad \forall t \in[0, T],
\end{aligned}
$$

with a constant $c>0$, independent of $h$ and $t$. Then, employing the Gronwall lemma, we find a constant $c>0$, independent of $h$ and $t$, such that

$$
\begin{aligned}
& \left\|\boldsymbol{\Phi}_{h} \boldsymbol{E}(t)-\boldsymbol{E}_{h}(t)\right\|_{\boldsymbol{L}_{\epsilon}^{2}(\Omega)}+\left\|\boldsymbol{\Pi}_{h} \boldsymbol{B}(t)-\boldsymbol{B}_{h}(t)\right\|_{\boldsymbol{L}_{1 / \mu}^{2}(\Omega)} \\
& \quad \leq c h^{s} \quad \forall h>0 \text { and all } t \in[0, T] .
\end{aligned}
$$

On the other hand, by Assumption 5.3, Lemma 5.2, and (5.4)-(5.5), there is a constant $c>0$, independent of $h$ and $t$, such that

$$
\left\{\begin{aligned}
\left\|\boldsymbol{\Phi}_{h} \boldsymbol{E}(t)-\boldsymbol{E}(t)\right\|_{\boldsymbol{L}_{\epsilon}^{2}(\Omega)} & \leq c h^{s}\|\boldsymbol{E}(t)\|_{\boldsymbol{H}_{0}^{s}(\mathbf{c u r l})} & & \forall h>0 \text { and all } t \in[0, T], \\
\left\|\boldsymbol{\Pi}_{h} \boldsymbol{B}(t)-\boldsymbol{B}(t)\right\|_{\boldsymbol{L}_{1 / \mu}^{2}(\Omega)} & \leq c h^{s}\|\boldsymbol{B}(t)\|_{\boldsymbol{H}^{s}(\Omega)} & & \forall h>0 \text { and all } t \in[0, T] .
\end{aligned}\right.
$$

The assertion follows now from (5.10) and (5.11). 
Remark 5.5. A fully discrete approximation of (VI) is obtained, for instance, by employing the implicit Euler method in $\left(\mathrm{VI}_{h}\right)$. More precisely, introducing the time step $\Delta t=\frac{T}{N}$, with $N \in \mathbb{N}$, we consider an equidistant partition of the interval $[0, T]$ as $0=t_{1}<t_{2}<\cdots<t_{N}=T$, where $t_{n}=n \Delta t$ for all $n=0, \ldots, N$. Then, applying the implicit backward Euler method to the semidiscrete Ritz-Galerkin approximation $\left(\mathrm{VI}_{h}\right)$, we arrive at the following fully discrete scheme:

$\left(\mathrm{VI}_{h, \Delta t}\right) \quad\left\{\begin{array}{l}\int_{\Omega} \epsilon \frac{\boldsymbol{E}_{h}^{n}-\boldsymbol{E}_{h}^{n-1}}{\Delta t} \cdot\left(\boldsymbol{v}_{h}-\boldsymbol{E}_{h}^{n}\right)+\mu^{-1} \frac{\boldsymbol{B}_{h}^{n}-\boldsymbol{B}_{h}^{n-1}}{\Delta t} \cdot\left(\boldsymbol{w}_{h}-\boldsymbol{B}_{h}^{n}\right) d x \\ +\int_{\Omega} \mu^{-1}\left(\operatorname{curl} \boldsymbol{E}_{h}^{n} \cdot\left(\boldsymbol{w}_{h}-\boldsymbol{B}_{h}^{n}\right)-\boldsymbol{B}_{h}^{n} \cdot \operatorname{curl}\left(\boldsymbol{v}_{h}-\boldsymbol{E}_{h}^{n}\right)\right) d x \\ +\varphi\left(\boldsymbol{v}_{h}\right)-\varphi\left(\boldsymbol{E}_{h}^{n}\right) \geq \int_{\Omega} \mathbf{f}(n \Delta t) \cdot\left(\boldsymbol{v}_{h}-\boldsymbol{E}_{h}^{n}\right) d x \quad \forall(\boldsymbol{v}, \boldsymbol{w}) \in \boldsymbol{V}_{h} \times \boldsymbol{W}_{h}, \\ n=1, \ldots, N, \quad\left(\boldsymbol{E}_{h}^{0}, \boldsymbol{B}_{h}^{0}\right)=\left(\boldsymbol{E}_{0 h}, \boldsymbol{B}_{0 h}\right) .\end{array}\right.$

Our future goal is to examine the fully discrete finite element approximations of (VI) such as $\left(\mathrm{VI}_{h, \Delta t}\right)$. While various implicit and explicit fully discrete schemata are available for parabolic $H^{1}(\Omega)$-type variational inequalities (see, e.g., [16]), we are only aware of the work by Elliott and Kashima [13] on the fully discrete numerical analysis for a parabolic Maxwell variational inequality of the first kind. Their results and [22] serve as an important basis for our future investigation.

\section{Appendix A.}

Lemma A.1. Let $\boldsymbol{u} \in W^{1,1}\left((0, T), \boldsymbol{L}_{\epsilon}^{2}(\Omega)\right)$. Then, the mapping $t \mapsto\|\boldsymbol{u}(t)\|_{\boldsymbol{L}_{\epsilon}^{2}(\Omega)}^{2}$ is of class $W^{1,1}(0, T)$ and

$$
\frac{d}{d t}\|\boldsymbol{u}(t)\|_{\boldsymbol{L}_{\epsilon}^{2}(\Omega)}^{2}=2 \int_{\Omega} \epsilon \boldsymbol{u}^{\prime}(t) \cdot \boldsymbol{u}(t) d x \quad \text { for a.e. } t \in(0, T) .
$$

Proof. Since $\mathcal{C}^{\infty}\left([0, T], \boldsymbol{L}_{\epsilon}^{2}(\Omega)\right)$ is dense in $W^{1,1}\left((0, T), \boldsymbol{L}_{\epsilon}^{2}(\Omega)\right)$, there is a sequence $\left\{\boldsymbol{u}_{n}\right\}_{n=1}^{\infty} \subset \mathcal{C}^{\infty}\left([0, T], \boldsymbol{L}_{\epsilon}^{2}(\Omega)\right)$ such that

$$
\lim _{n \rightarrow \infty}\left\|\boldsymbol{u}_{n}-\boldsymbol{u}\right\|_{W^{1,1}\left((0, T), \boldsymbol{L}_{\epsilon}^{2}(\Omega)\right)}=0 .
$$

As $\left\{\boldsymbol{u}_{n}\right\}_{n=1}^{\infty} \subset \mathcal{C}^{\infty}\left([0, T], \boldsymbol{L}_{\epsilon}^{2}(\Omega)\right)$, it holds that

$$
\left\|\boldsymbol{u}_{n}(t)\right\|_{\boldsymbol{L}_{\epsilon}^{2}(\Omega)}^{2}-\left\|\boldsymbol{u}_{n}(0)\right\|_{\boldsymbol{L}_{\epsilon}^{2}(\Omega)}^{2}=2 \int_{0}^{t} \int_{\Omega} \epsilon \boldsymbol{u}_{n}^{\prime}(s) \cdot \boldsymbol{u}_{n}(s) d x d s \quad \forall t \in[0, T], \quad \forall n \in \mathbb{N} .
$$

On the other hand, the embedding $W^{1,1}\left((0, T), \boldsymbol{L}_{\epsilon}^{2}(\Omega)\right) \hookrightarrow \mathcal{C}\left([0, T], \boldsymbol{L}_{\epsilon}^{2}(\Omega)\right)$ implies (possibly after a modification on a subset of $[0, T]$ with zero measure) that $\boldsymbol{u} \in$ $\mathcal{C}\left([0, T], \boldsymbol{L}_{\epsilon}^{2}(\Omega)\right)$, and there is a constant $c>0$, independent of $n$, such that

$$
\left\|\boldsymbol{u}_{n}-\boldsymbol{u}\right\|_{\mathcal{C}\left([0, T], \boldsymbol{L}_{\epsilon}^{2}(\Omega)\right)} \leq c\left\|\boldsymbol{u}_{n}-\boldsymbol{u}\right\|_{W^{1,1}\left((0, T), \boldsymbol{L}_{\epsilon}^{2}(\Omega)\right)} \quad \forall n \in \mathbb{N} .
$$

Consequently, (A.1) implies that

$$
\lim _{n \rightarrow \infty}\left\|\boldsymbol{u}_{n}-\boldsymbol{u}\right\|_{\mathcal{C}\left([0, T], \boldsymbol{L}_{\epsilon}^{2}(\Omega)\right)}=0 .
$$


Now, in view of (A.1) and (A.3), passing to the limit $n \rightarrow \infty$ in (A.2) yields that

$$
\|\boldsymbol{u}(t)\|_{\boldsymbol{L}_{\epsilon}^{2}(\Omega)}^{2}-\|\boldsymbol{u}(0)\|_{\boldsymbol{L}_{\epsilon}^{2}(\Omega)}^{2}=2 \int_{0}^{t} \int_{\Omega} \epsilon \boldsymbol{u}^{\prime}(s) \cdot \boldsymbol{u}(s) d x d s=\int_{0}^{t} g(s) d s \quad \forall t \in[0, T]
$$

with $g \in L^{1}(0, T), g(s):=2\left(\boldsymbol{u}^{\prime}(s), \boldsymbol{u}(s)\right)_{\boldsymbol{L}_{\epsilon}^{2}(\Omega)}$. By a classical result, it follows from (A.4) that the mapping $t \mapsto\|\boldsymbol{u}(t)\|_{\boldsymbol{L}_{\epsilon}^{2}(\Omega)}^{2}$ is of class $W^{1,1}(0, T)$ and

$$
\frac{d}{d t}\|\boldsymbol{u}(t)\|_{\boldsymbol{L}_{\epsilon}^{2}(\Omega)}^{2}=g(t)=2 \int_{\Omega} \epsilon \boldsymbol{u}^{\prime}(t) \cdot \boldsymbol{u}(t) d x \quad \text { for a.e. } t \in(0, T) .
$$

\section{REFERENCES}

[1] A. Alonso And A. Valli, Eddy Current Approximation of Maxwell Equations: Theory Algorithms and Applications, Springer, New York, 2010.

[2] C. Amrouche, C. Bernardi, M. Dauge, and V. Girault, Vector potentials in threedimensional non-smooth domains, Math. Methods Appl. Sci., 21 (1998), pp. 823-864.

[3] V. Barbu, Optimal Control of Variational Inequalities, Pitman (Advanced Publishing Program), Boston, MA, 1984.

[4] J. W. Barrett and L. Prigozhin, Dual formulations in critical state problems, Interfaces Free Bound., 8 (2006), pp. 349-370.

[5] J. W. Barrett And L. Prigozhin, Existence and approximation of a mixed formulation for thin film magnetization problems in superconductivity, Math. Models Methods Appl. Sci., 24 (2014), pp. 991-1015.

[6] J. W. Barrett And L. Prigozhin, Sandpiles and superconductors: Nonconforming linear finite element approximations for mixed formulations of quasi-variational inequalities, IMA J. Numer. Anal., 35 (2015), pp. 1-38.

[7] C. P. Bean, Magnetization of hard superconductors, Phys. Rev. Lett., 8 (1962), pp. 250-253.

[8] C. P. Bean, Magnetization of high-field superconductors, Rev. Modern Phys., 36 (1964), pp. 31-39.

[9] A. Bossavit, Numerical modelling of superconductors in three dimensions: A model and a finite element method, IEEE Trans. Magnetics, 30 (1994), pp. 3363-3366.

[10] S. J. Chapman, A hierarchy of models for type-II superconductors, SIAM Rev., 42 (2000), pp. $555-598$

[11] Z.-M. Chen, K.-H. Hoffmann, and J. Liang, On a nonstationary Ginzburg-Landau superconductivity model, Math. Methods Appl. Sci., 16 (1993), pp. 855-875.

[12] Q. Du, M. D. Gunzburger, And J. S. Peterson, Analysis and approximation of the GinzburgLandau model of superconductivity, SIAM Rev., 34 (1992), pp. 54-81.

[13] C. M. Elliott and Y. Kashima, A finite-element analysis of critical-state models for type-II superconductivity in 3D, IMA J. Numer. Anal., 27 (2007), pp. 293-331.

[14] C. M. Elliott, D. Kay, And V. Styles, A finite element approximation of a variational inequality formulation of Bean's model for superconductivity, SIAM J. Numer. Anal., 42 (2004), pp. 1324-1341.

[15] V. Girault and P. Raviart, Finite Element Methods for Navier-Stokes Equations, SpringerVerlag, Berlin, 1986.

[16] R. Glowinski, Numerical Methods for Nonlinear Variational Problems, Scientific Computation, Springer-Verlag, Berlin, 2008. Reprint of the 1984 original.

[17] R. Hiptmair, Finite elements in computational electromagnetism, Acta Numer., 11 (2002), pp. 237-339.

[18] F. Jochmann, On a first-order hyperbolic system including Bean's model for superconductors with displacement current, J. Differential Equations, 246 (2009), pp. 2151-2191.

[19] F. Jochmann, A variational inequality in Bean's model for superconductors with displacement current, Discrete Contin. Dyn. Syst., 25 (2009), pp. 545-565.

[20] F. Jochmann, Well-posedness for Bean's critical state model with displacement current, J. Math. Anal. Appl., 362 (2010), pp. 505-513.

[21] Y. B. Kim, C. F. Hempstead, and A. R. Strnad, Magnetization and critical supercurrents, Phys. Rev., 129 (1963), pp. 528-535. 
[22] Ch. G. Makridakis and P. Monk, Time-discrete finite element schemes for Maxwell's equations, RAIRO Modél. Math. Anal. Numér., 29 (1995), pp. 171-197.

[23] P. Monk, Finite Element Methods for Maxwell's Equations, Clarendon, Oxford, UK, 2003.

[24] J. C. NÉDÉLEC, Mixed finite elements in $\mathbb{R}^{3}$, Numer. Math., 35 (1980), pp. 315-341.

[25] L. Prigozhin, The Bean model in superconductivity: Variational formulation and numerical solution, J. Comput. Phys., 129 (1996), pp. 190-200.

[26] L. Prigozhin, On the Bean critical-state model in superconductivity, European J. Appl. Math., 7 (1996), pp. 237-247.

[27] I. YOUSEPT, Optimal control of Maxwell's equations with regularized state constraints, Comput. Optim. Appl., 52 (2012), pp. 559-581.

[28] I. Yousept, Optimal control of quasilinear $\boldsymbol{H}$ (curl)-elliptic partial differential equations in magnetostatic field problems, SIAM J. Control Optim., 51 (2013), pp. 3624-3651.

[29] I. Yousept, Optimal control of non-smooth hyperbolic evolution Maxwell equations in type-II superconductivity, SIAM J. Control Optim., 55 (2017), pp. 2305-2332. 